TRANSACTIONS OF THE

AMERICAN MATHEMATICAL SOCIETY

Volume 354, Number 8 , Pages 3349-3378

S 0002-9947(02)02980-X

Article electronically published on April 1, 2002

\title{
HOPF MODULES AND THE DOUBLE OF A QUASI-HOPF ALGEBRA
}

\author{
PETER SCHAUENBURG
}

\begin{abstract}
We give a different proof for a structure theorem of Hausser and Nill on Hopf modules over quasi-Hopf algebras. We extend the structure theorem to a classification of two-sided two-cosided Hopf modules by YetterDrinfeld modules, which can be defined in two rather different manners for the quasi-Hopf case. The category equivalence between Hopf modules and Yetter-Drinfeld modules leads to a new construction of the Drinfeld double of a quasi-Hopf algebra, as proposed by Majid and constructed by Hausser and Nill.
\end{abstract}

\section{INTRODUCTION}

Drinfeld's quantum double construction has established itself as an important tool in the theory of Hopf algebras. In particular, it furnishes nontrivial examples of quasitriangular, non-commutative and non-cocommutative Hopf algebras, and the double $D(H)$ can be used to study the original Hopf algebra $H$. On the categorical level, the Drinfeld double can be explained by the construction of the center of a monoidal category; this is a braided monoidal category $\mathcal{Z}(\mathcal{C})$ that can be constructed in a natural way from any monoidal category $\mathcal{C}$. One has ${ }_{D(H)} \mathcal{M} \cong \mathcal{Z}\left({ }_{H} \mathcal{M}\right)$ for any finite-dimensional Hopf algebra $H$. Yetter-Drinfeld modules can be seen as a convenient intermediate step in this equivalence; one has $\mathcal{Z}\left({ }_{H} \mathcal{M}\right) \cong{ }_{H} \mathcal{Y} \mathcal{D}^{H}$ (even if $H$ is infinite-dimensional, which makes YetterDrinfeld modules interesting beyond situations where the quantum double can be constructed), and the equivalence ${ }_{H} \mathcal{Y} \mathcal{D}^{H} \cong{ }_{D(H)} \mathcal{M}$ is a very easy step if $H$ is finite-dimensional. We refer to Kassel's book [7] for a complete account.

Quasi-Hopf algebras were introduced by Drinfeld in 2. Although they arise there primarily from the deformation theoretical treatment of ordinary Hopf algebras, the conceptual categorical explanation of their axioms is already discussed in detail in [2]: The definition ensures that the category of finite-dimensional modules over a quasi-Hopf algebra $H$ is a monoidal category with duality. The tensor product in the module category is the tensor product over the base field $k$, and $H$ has a comultiplication that allows one to endow this tensor product with a module structure, and an invertible element $\phi \in H \otimes H \otimes H$ responsible for the associativity of tensor products in the module category. The key difference compared to an

Received by the editors April 10, 2001 and, in revised form, November 13, 2001.

2000 Mathematics Subject Classification. Primary 16W30.

Key words and phrases. Quasi-Hopf algebra, quantum double, Yetter-Drinfeld module, Hopf module. 
ordinary Hopf algebra is that comultiplication is no longer coassociative, but only so up to conjugation by $\phi$.

The first quantum double quasi-Hopf algebra appeared in a paper by Dijkgraaf, Pasquier, and Roche [1] namely, a certain variant $D^{\omega}(G)$ of the Drinfeld double of the group algebra $k G$ of a finite group, modified by a three-cocycle $\omega$ on $G$.

Majid [10] gave the following conceptual explanation of the double $D^{\omega}(G)$ : It is simply the Drinfeld double of a certain quasi-Hopf algebra, namely the dual group algebra $k^{G}$, with a nontrivial quasi-Hopf structure $\omega \in k^{G} \otimes k^{G} \otimes k^{G}$ that identifies with the group cocycle $\omega$. Of course, one has to explain now what the Drinfeld double of a quasi-Hopf algebra is, and Majid announces a construction. Note that the generalization from the Hopf to the quasi-Hopf case cannot be expected to be trivial; after all, the double of a Hopf algebra $H$ is modelled on $H \otimes H^{*}$, with $H$ and $H^{*}$ as subalgebras. But if $H$ is just a quasi-Hopf algebra, then $H^{*}$ is not an associative algebra, so one is at a loss looking for an associative algebra structure on $H \otimes H^{*}$. Majid [10] explains why the construction should work; the module category over the Drinfeld double $D(H)$ of an ordinary Hopf algebra $H$ is equivalent to the center of the category of $H$-modules. The center construction is a purely categorical procedure assigning a braided monoidal category to any monoidal category; of course it can be applied to the category of $H$-modules also when $H$ is just a quasi-Hopf algebra. The result should be the module category for another quasi-Hopf algebra $D(H)$, the Drinfeld double of $H$ - by the general 'reconstruction' principles by which every reasonably nice monoidal category has to be the representation category of some quasi-Hopf algebra. Next, of course, one would like to know the double explicitly. Majid claims to show that $D(H)$ can, once again, be modelled on the tensor product $H \otimes H^{*}$. As we shall try to indicate at the end of Section 9 there is some doubt that the reasoning in [10] is complete here. However, Hausser and Nill [3, 4] have indeed shown by explicit calculations that the double can be realized on $H \otimes H^{*}$, although perhaps not quite in the way anticipated in [10]. The basic idea in [10] is that the center of the category ${ }_{H} \mathcal{M}$ of left $H$-modules is equivalent to the category ${ }_{H} \mathcal{Y D}^{H}$ of Yetter-Drinfeld $H$-modules. The latter (which are defined in [10]) are modules equipped with a certain coaction of $H$ (which would be a comodule structure in the ordinary Hopf case) satisfying two compatibility conditions. Thus, since a Yetter-Drinfeld module is given by an action of $H$ and an action of $H^{*}$ satisfying some commutation relations, one may surmise that it can equivalently be given by an action of $H \otimes H^{*}$, where the latter is endowed with a suitable multiplication that can be read off from the compatibility conditions. The additional idea introduced by Hausser and Nill to make this work is the realization that there are two rather different, but equivalent, variants of Yetter-Drinfeld modules in the quasi-Hopf case. (Actually the terminology in [3, 4] is quite different, and we will not go into details on how exactly to relate it to ours.) One version (called a coherent $\delta$-implementer by Hausser and Nill) is better suited to describing multiplication in the Drinfeld double $D(H)=H \otimes H^{*}$, while the other (called a coherent $\Delta$-flip operator) is better suited to describing comultiplication, antipode, and quasitriangular structure.

In the present paper we will be dealing with another characterization of YetterDrinfeld modules (hence the Drinfeld double and the center), namely the equivalence of the category of Yetter-Drinfeld modules with the category ${ }_{H}^{H} \mathcal{M}_{H}^{H}$ of Hopf modules; this equivalence originally arose in work of Woronowicz [17] on differential calculi on quantum groups, and was developed for Hopf algebras in [13. We will 
extend it to the quasi-Hopf algebra setting, and utilize it in the construction of the Drinfeld double.

Hopf modules over quasi-Hopf algebras were introduced by Hausser and Nill [5] to develop an integral theory for quasi-Hopf algebras. Note that the definition of Hopf modules for quasi-Hopf algebras requires a little thought, since a quasiHopf algebra is not a coalgebra, hence there is no immediate notion of comodule. But one can define ${ }_{H} \mathcal{M}_{H}^{H}$ as the category of $H$-comodules within the monoidal category ${ }_{H} \mathcal{M}_{H}$ of $H$-bimodules. With this categorical description comes an easy supply of such Hopf modules, namely the cofree $H$-comodules $P \otimes H$ generated by $P \in{ }_{H} \mathcal{M}_{H}$. In particular, one can apply this to one-sided $H$-modules. Now the structure theorem of Hausser and Nill, for which we shall give a new proof in Section 3, asserts that every Hopf module in ${ }_{H} \mathcal{M}_{H}^{H}$ is of the form $V \otimes H$ for some $V \in{ }_{H} \mathcal{M}$, provided $H$ is a quasi-Hopf algebra. The equivalence ${ }_{H} \mathcal{M} \cong{ }_{H} \mathcal{M}_{H}^{H}$ thus established is actually a monoidal equivalence, when we endow ${ }_{H} \mathcal{M}_{H}^{H}$ with the monoidal category structure defined by the tensor product over $H$.

We define the Hopf module category ${ }_{H}^{H} \mathcal{M}_{H}^{H}$ to be the category of $H$-H-bicomodules in the monoidal category ${ }_{H} \mathcal{M}_{H}$. There are two natural monoidal category structures on ${ }_{H}^{H} \mathcal{M}_{H}^{H}$, namely tensor product and cotensor product over $H$, which we prove to be isomorphic if $H$ is a quasi-Hopf algebra. Now by the structure theorem of Hausser and Nill, understanding the structure of Hopf modules in ${ }_{H}^{H} \mathcal{M}_{H}^{H}$ amounts to describing all the additional left $H$-coactions making $V \otimes H \in{ }_{H} \mathcal{M}_{H}^{H}$ an object of ${ }_{H}^{H} \mathcal{M}_{H}^{H}$, for $V \in{ }_{H} \mathcal{M}$. We attack this problem in two different ways, arriving at two different notions of Yetter-Drinfeld modules. We call Yetter-Drinfeld modules of the first kind the ones appearing (without a name) in Majid's paper [10. We study Yetter-Drinfeld modules of the second kind first, in Section 5. They arise from the following approach: A left $H$-coaction making $M=V \otimes H$ an object in ${ }_{H}^{H} \mathcal{M}_{H}^{H}$ is by definition a morphism $\Lambda: M \rightarrow H \otimes M$ in ${ }_{H} \mathcal{M}_{H}^{H}$. The latter category is equivalent to ${ }_{H} \mathcal{M}$, so we can send $\Lambda$ through the category equivalence to give, as it turns out, a certain map $\lambda^{\prime}: V \rightarrow H \otimes V$, which makes $V$ a Yetter-Drinfeld module of the second kind. The definition of the latter can be made to look very simple. In fact, using terminology of Pareigis [12, one may say that a Yetter-Drinfeld module of the second kind is simply an $H$-comodule - however, the coalgebra $H$ is situated in the monoidal category ${ }_{H} \mathcal{M}_{H}$, whereas its comodule $V$ is situated in a different category ${ }_{H} \mathcal{M}$, on which the category ${ }_{H} \mathcal{M}_{H}$ acts in a nontrivial way. This action is described explicitly in Section 4 .

Yetter-Drinfeld modules of the second kind are well-suited to the description of the Drinfeld double of $H$ as an associative algebra, which we give in Section 6 Although the formula for multiplication is quite complicated, it is rather easy to verify that it is associative, thanks to the conceptual nature of the Hopf module category, which supplies us with 'enough' modules, without the necessity of performing large calculations.

We study Yetter-Drinfeld modules of the first kind in Section 8 . These objects were introduced (with different conventions) by Majid [10] to describe the center of the module category ${ }_{H} \mathcal{M}$. We repeat this description, and we show that YetterDrinfeld modules of the first kind can also be used to classify Hopf modules in ${ }_{H}^{H} \mathcal{M}_{H}^{H}$. This is based on the following approach to Hopf module structures: By the structure theorem of Hausser and Nill, any $M \in{ }_{H} \mathcal{M}_{H}^{H}$ is the cofree right $H$-comodule over some $V \in{ }_{H} \mathcal{M}$, within the category ${ }_{H} \mathcal{M}_{H}$. The problem of describing all bicomodule structures on a cofree right $H$-comodule was essentially 
treated by Tambara; strictly speaking only the bimodule structures on a free right module over a $k$-algebra were treated in [16], but the generalization to monoidal categories, hence also to comodules in monoidal categories, is a trivial step. Tambara's description involves a flipping map $\sigma: V \otimes H \rightarrow H \otimes V$, and it yields a category equivalence of ${ }_{H}^{H} \mathcal{M}_{H}^{H}$ with the center of ${ }_{H} \mathcal{M}$, or the category of YetterDrinfeld modules of the first kind, if $H$ is a quasi-Hopf algebra. In particular, we also obtain a category equivalence between Yetter-Drinfeld modules of the first and second kinds, which parallels the correspondence between the $\delta$-implementers and $\Delta$-flip operators of Hausser and Nill.

Yetter-Drinfeld modules of the first kind are well suited to describing the quasitriangular quasi-Hopf algebra structure of the double $D(H)$, which we do in Section 9

Summing up, we think that the correspondence between Hopf modules and Yetter-Drinfeld modules may be an interesting fact in itself. While the construction of the quantum double is not new, we believe that some additional insight can be gained by treating it through this correspondence. In particular, it is our feeling that this approach allows us to replace some explicit and ad hoc calculations with somewhat more conceptual reasoning.

\section{Preliminaries}

Throughout the paper we work over a base field $k$. If nothing else is indicated, vector spaces, algebras, etc. are over $k, \otimes$ means $\otimes_{k}$, Hom means $\operatorname{Hom}_{k}$, and so forth. For any $k$-algebra we write ${ }_{A} \mathcal{M}$ for the category of left $A$-modules, with obvious variants for right modules and bimodules. $A^{\text {op }}$ denotes the opposite algebra. We write $a-\varphi$ and $\varphi<a$ for the canonical left and right actions of an algebra $A$ on its dual space $A^{*}$. If $A, B$ are algebras, and $M \in \mathcal{M}_{A}, N \in{ }_{A} \mathcal{M}_{B}$, and $P \in{ }_{B} \mathcal{M}$ are (bi-)modules, we will take the liberty to pretend that the canonical isomorphisms $\left(M \otimes_{A} N\right) \otimes_{B} P \cong M \otimes_{A}\left(N \otimes_{B} P\right)$ and $A \otimes_{A} N \cong N \cong N \otimes_{B} B$ are identities.

2.1. Monoidal categories. A monoidal category $\mathcal{C}=(\mathcal{C}, \otimes, \Phi, I, \lambda, \rho)$ consists of a category $\mathcal{C}$, a functor $\otimes: \mathcal{C} \times \mathcal{C} \rightarrow \mathcal{C}$, a "unit" object $I$ and natural isomorphisms $\Phi=\Phi_{X Y Z}:(X \otimes Y) \otimes Z \rightarrow X \otimes(Y \otimes Z), \lambda: I \otimes X \rightarrow X$ and $\rho: X \otimes I \rightarrow X$, which are required to be 'coherent'. We refer to [6, 7] (where monoidal categories are called tensor categories) for precise definitions and background information. We shall always assume that the morphisms $\lambda$ and $\rho$ are identities; when $\Phi$ is the identity as well, $\mathcal{C}$ is called strict. Coherence is expressed in Mac Lane's pentagon

$$
\left(W \otimes \Phi_{X Y Z}\right) \Phi_{W, X \otimes Y, Z}\left(\Phi_{W X Y} \otimes Z\right)=\Phi_{W, X, Y \otimes Z} \Phi_{W \otimes X, Y, Z},
$$

where both sides are morphisms $((W \otimes X) \otimes Y) \otimes Z \rightarrow W \otimes(X \otimes(Y \otimes Z))$, and the condition $\Phi_{X I Y}=\operatorname{id}_{X Y}$, which also implies $\Phi_{X Y I}=\Phi_{I X Y}=\operatorname{id}_{X Y}$. The key consequence of coherence is that all formal diagrams composed of associator morphisms commute.

We define an (incoherent) tensor functor

$$
(\mathcal{F}, \xi):(\mathcal{C}, \otimes, \Phi, I) \rightarrow\left(\mathcal{D}, \otimes, \Phi^{\prime}, I^{\prime}\right)
$$

to consist of a functor $\mathcal{F}$ satisfying $\mathcal{F}(I)=I^{\prime}$, and a natural isomorphism $\xi: \mathcal{F}(X) \otimes$ $\mathcal{F}(Y) \rightarrow \mathcal{F}(X \otimes Y)$ such that $\xi_{X I}=\xi_{I X}=\operatorname{id}_{\mathcal{F}(X)}$. If $\xi$ is the identity, we say that 
$\mathcal{F}$ is strict. We define a monoidal functor to be a tensor functor that is coherent in the sense that the diagrams

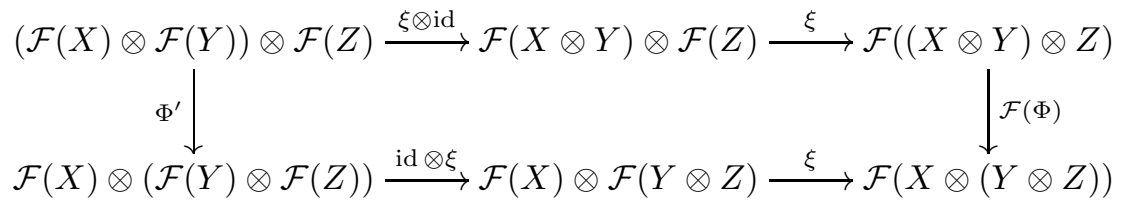

commute. (Warning: In [7 monoidal functors are called tensor functors.)

A nice way of expressing coherence is to say [7, XI.5] that every monoidal category is equivalent, via a monoidal functor, to a strict one. Since monoidal functors can be used to transport notions expressible in the language of monoidal categories, this leads to the slogan that, to prove a general fact on monoidal categories, it is always sufficient to treat the strict case. Before adopting this widespread usage of coherence, the author would like to acknowledge that he knows of no general metatheorem that would, once and for all, settle the question of what types of facts this reasoning can be applied to.

Let $\mathcal{C}$ be a monoidal category and $X \in \mathcal{C}$. A left dual of $X$ is a collection $\left(X^{\vee}, \mathrm{ev}, \mathrm{db}\right)$ in which $X^{\vee}$ is another object of $\mathcal{C}$, and ev : $X^{\vee} \otimes X \rightarrow I$ and db: $I \rightarrow$ $X \otimes X^{\vee}$ are morphisms (called evaluation and coevaluation, respectively) such that

$$
\begin{gathered}
\left(X \stackrel{\mathrm{db} \otimes X}{\longrightarrow}\left(X \otimes X^{\vee}\right) \otimes X \stackrel{\Phi}{\longrightarrow} X \otimes\left(X^{\vee} \otimes X\right) \stackrel{X \otimes \mathrm{ev}}{\longrightarrow} X\right)=\mathrm{id}_{X}, \\
\left(X^{\vee} \stackrel{X^{\vee} \otimes \mathrm{db}}{\longrightarrow} X^{\vee} \otimes\left(X \otimes X^{\vee}\right) \stackrel{\Phi^{-1}}{\longrightarrow}\left(X^{\vee} \otimes X\right) \otimes X^{\vee} \stackrel{\mathrm{ev} \otimes X^{\vee}}{\longrightarrow} X^{\vee}\right)=\mathrm{id}_{X} \vee
\end{gathered}
$$

If every object of $\mathcal{C}$ has a left dual, we say that $\mathcal{C}$ is (left) rigid. In this case $X \mapsto X^{\vee}$ can be made into a contravariant functor in a natural way. Moreover, there exists a unique natural isomorphism $\mathrm{D}: Y^{\vee} \otimes X^{\vee} \rightarrow(X \otimes Y)^{\vee}$ such that

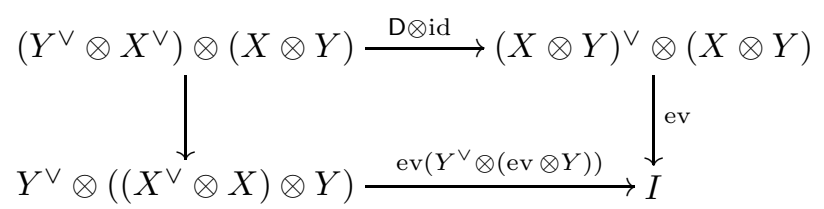

commutes.

Right duals and right rigidity are defined by switching sides.

2.2. Coalgebras in monoidal categories. Assume given a monoidal category $\mathcal{C}$. An algebra in $\mathcal{C}$ is a pair $(A, \nabla)$ in which $A$ is an object of $\mathcal{C}$, and $\nabla: A \otimes A \rightarrow A$ is a morphism, called multiplication, such that

$$
\nabla(\nabla \otimes A)=\nabla(A \otimes \nabla) \Phi:(A \otimes A) \otimes A \rightarrow A,
$$

i.e., multiplication is associative, and there exists a unit morphism $\eta: I \rightarrow A$ such that $\nabla(A \otimes \eta)=\operatorname{id}_{A}=\nabla(\eta \otimes A)$. A left $A$-module over an algebra $A$ in $\mathcal{C}$ is an object $M$ in $\mathcal{C}$ with a morphism $\mu: A \otimes M \rightarrow M$ such that $\mu(\eta \otimes M)=\operatorname{id}_{M}$ and

$$
\mu(\nabla \otimes M)=\mu(A \otimes \mu) \Phi:(A \otimes A) \otimes M \rightarrow M .
$$

Many elementary facts of ring and module theory can be carried over to this generalized notion of algebra (which, of course, contains $k$-algebra theory as the special case $\mathcal{C}=\mathcal{M}_{k}$ ). More details can be found in [11]. It is convenient to utilize the coherence result by which any base category $\mathcal{C}$ is equivalent as a monoidal category 
to a strict one - hence, if one proves general results on algebras and modules, one can always assume that the base category is strict.

Since algebras and modules are defined in entirely categorical terms, the general theory includes a theory of coalgebras and comodules as a special case - one just has to replace the base category by its opposite to switch from algebras to coalgebras. In the following we will spell out some facts of such a generalized coalgebra theory, which shall be used freely in the paper. We will not repeat the definition of coalgebra and comodule, dual to those of algebra and module. We denote the category of left (right) $C$-comodules in $\mathcal{C}$ by ${ }^{C} \mathcal{C}$ and $\mathcal{C}^{C}$, respectively. If $C$ is a coalgebra, $M$ a right $C$-comodule, and $X$ any object of $\mathcal{C}$, then $X \otimes M$ is a $C$-comodule with comodule structure

$$
X \otimes M \stackrel{X \otimes \rho}{\longrightarrow} X \otimes(M \otimes C) \stackrel{\Phi^{-1}}{\longrightarrow}(X \otimes M) \otimes C,
$$

where $\rho$ is the comodule structure of $M$. As a special case, $X \otimes C$ is a right $C$-comodule, and actually the cofree right $C$-comodule over $X$, in the sense that we have a bijection $\mathcal{C}(M, X) \ni f \mapsto(f \otimes C) \rho \in \mathcal{C}^{C}(M, X \otimes C)$ with inverse $F \mapsto(X \otimes \varepsilon) F$, so that the functor $\mathcal{C} \ni X \mapsto X \otimes C \in \mathcal{C}^{C}$ is right adjoint to the underlying functor $\mathcal{C}^{C} \rightarrow \mathcal{C}$. In particular, the morphism $p: X \otimes \varepsilon: X \otimes C \rightarrow X$ satisfies $(p \otimes C) \rho_{X \otimes C}=\operatorname{id}_{X \otimes C}$. We note for later use that, if $Y$ is another object of $\mathcal{C}$, the $C$-comodule structure $\rho$ of $Y \otimes(X \otimes C)$ satisfies

$$
\left(Y \otimes(X \otimes C) \stackrel{\rho}{\rightarrow}(Y \otimes(X \otimes C)) \otimes C \stackrel{\varepsilon_{3}}{\longrightarrow}(Y \otimes X) \otimes C\right)=\Phi^{-1},
$$

where $\varepsilon_{3}:=(Y \otimes(X \otimes \varepsilon)) \otimes C$, simply since this is trivial if $\mathcal{C}$ is strict.

A $C$-D-bicomodule for coalgebras $C, D$ in $\mathcal{C}$ is an object $M$ with morphisms $\lambda: M \rightarrow C \otimes M$ and $\rho: M \rightarrow M \otimes D$ making it a left and right comodule, respectively, and satisfying

$$
(C \otimes \rho) \lambda=\Phi(\lambda \otimes D) \rho: M \rightarrow C \otimes(M \otimes D) .
$$

We denote the category of $C$-D-bicomodules in $\mathcal{C}$ by ${ }^{C} \mathcal{C}^{D}$. If $M \in{ }^{C} \mathcal{C}$ and $N \in \mathcal{C}^{D}$, then $M \otimes N \in{ }^{C} \mathcal{C}^{D}$ with the right $D$-comodule structure as introduced above, and the analogous left $C$-comodule structure. In particular, if $X \in{ }^{C} \mathcal{C}$, then the cofree right $D$-comodule over $X$ is a $C$ - $D$-bicomodule; thus we have a right adjoint to the underlying functor ${ }^{C} \mathcal{C}^{D} \rightarrow{ }^{C} \mathcal{C}$.

Assume from now on that the category $\mathcal{C}$ has equalizers, and that the tensor product in $\mathcal{C}$ preserves equalizers in each variable. By definition the cotensor product of $M \in \mathcal{C}^{C}$ and $N \in{ }^{C} \mathcal{C}$ is defined as the equalizer

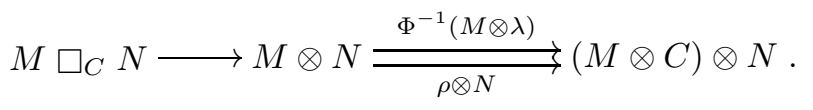

If $M \in{ }^{B} \mathcal{C}^{C}$ and $N \in{ }^{C} \mathcal{C}^{D}$, then $M \square_{C} N \in{ }^{B} \mathcal{C}^{D}$ as a sub-bicomodule of $M \otimes N$. In particular, the category ${ }^{C} \mathcal{C}^{C}$ is a monoidal category with respect to $\square_{C}$; the associators are induced by the associators in $\mathcal{C}$.

If $M=X \otimes C$ is the cofree right $C$-comodule over $X$, then we have $M \square_{C} N \cong$ $X \otimes N$, with the isomorphism induced by $(M \otimes \varepsilon) \otimes N$, and its inverse induced by

$$
X \otimes N \stackrel{X \otimes \lambda}{\longrightarrow} X \otimes(C \otimes N) \stackrel{\Phi^{-1}}{\longrightarrow}(X \otimes C) \otimes N .
$$


2.3. Quasi-Hopf algebras. A quasibialgebra $H=(H, \Delta, \varepsilon, \phi)$ consists of an algebra $H$, algebra maps $\Delta: H \rightarrow H \otimes H$ and $\varepsilon: H \rightarrow k$, and an invertible element $\phi \in H^{\otimes 3}$, the associator, such that

$$
\begin{gathered}
(\varepsilon \otimes H) \Delta(h)=h=(H \otimes \varepsilon) \Delta(h), \\
(H \otimes \Delta) \Delta(h) \cdot \phi=\phi \cdot(\Delta \otimes H) \Delta(h), \\
(H \otimes H \otimes \Delta)(\phi) \cdot(\Delta \otimes H \otimes H)(\phi)=(1 \otimes \phi) \cdot(H \otimes \Delta \otimes H)(\phi) \cdot(\phi \otimes 1), \\
(H \otimes \varepsilon \otimes H)(\phi)=1
\end{gathered}
$$

hold for all $h \in H$, the equations being in $H, H \otimes H \otimes H, H^{\otimes 4}$, and $H^{\otimes 2}$, respectively. It follows that also $(\varepsilon \otimes H \otimes H)(\phi)=1=(H \otimes H \otimes \varepsilon)(\phi)$. We will write $\Delta(h)=: h_{(1)} \otimes h_{(2)}, \phi=\phi^{(1)} \otimes \phi^{(2)} \otimes \phi^{(3)}$, and $\phi^{-1}=\phi^{(-1)} \otimes \phi^{(-2)} \otimes \phi^{(-3)}$. When there is need to perform computations with several copies of $\phi$, we write $\phi=\tilde{\phi}^{(1)} \otimes \tilde{\phi}^{(2)} \otimes \tilde{\phi}^{(3)}$ for a second copy of $\phi$, and so forth. We will adopt a similar notation for the tensor factors of other elements of tensor products.

If $H$ is a quasibialgebra, then the category ${ }_{H} \mathcal{M}$ of left $H$-modules is a monoidal category in the following way: For $V, W \in{ }_{H} \mathcal{M}$ the tensor product $V \otimes W$ is an $H$ module by $h(v \otimes w)=h_{(1)} v \otimes h_{(2)} w$; this defines a bifunctor $\otimes:{ }_{H} \mathcal{M} \times{ }_{H} \mathcal{M} \rightarrow{ }_{H} \mathcal{M}$. The base ring $k$ is an $H$-module via $\varepsilon$. Then the canonical morphisms $V \cong V \otimes k \cong$ $k \otimes V$ are $H$-linear for $V \in{ }_{H} \mathcal{M}$. The map

$$
\Phi:(U \otimes V) \otimes W \ni u \otimes v \otimes w \mapsto \phi^{(1)} u \otimes \phi^{(2)} v \otimes \phi^{(3)} w \in U \otimes(V \otimes W)
$$

is $H$-linear as a consequence of (2.4), and makes Mac Lane's pentagon commute as a consequence of (2.5).

The monoidal category structure on ${ }_{H} \mathcal{M}$ is defined in such a way that the underlying functor $\mathcal{U}:{ }_{H} \mathcal{M} \rightarrow \mathcal{M}_{k}$ preserves tensor products and the unit object; that is, so that the underlying functor is a strict incoherent tensor functor. Conversely, if $H$ is an algebra such that ${ }_{H} \mathcal{M}$ is a monoidal category, and the underlying functor $\mathcal{U}:{ }_{H} \mathcal{M} \rightarrow \mathcal{M}_{k}$ is a strict incoherent tensor functor, then there is a unique quasibialgebra structure on $H$ inducing the given monoidal category structure on ${ }_{H} \mathcal{M}$.

If $(H, \phi)$ is a quasibialgebra, so is $\left(H^{\mathrm{op}}, \phi^{-1}\right)$, and if $(B, \psi)$ is another quasibialgebra, then $H \otimes B$ is a quasibialgebra with associator $\phi^{(1)} \otimes \psi^{(1)} \otimes \phi^{(2)} \otimes \psi^{(2)} \otimes$ $\phi^{(3)} \otimes \psi^{(3)}$.

A quasiantipode $(S, \alpha, \beta)$ for a quasibialgebra $H$ consists of an algebra antiautomorphism $S$ of $H$, and elements $\alpha, \beta \in H$, such that

$$
\begin{aligned}
S\left(h_{(1)}\right) \alpha h_{(2)} & =\varepsilon(h) \alpha, & h_{(1)} \beta S\left(h_{(2)}\right) & =\varepsilon(h) \beta, \\
\phi^{(1)} \beta S\left(\phi^{(2)}\right) \alpha \phi^{(3)} & =1, & S\left(\phi^{(-1)}\right) \alpha \phi^{(-2)} \beta \phi^{(-3)} & =1
\end{aligned}
$$

hold in $H$, for $h \in H$. A quasi-Hopf algebra is a quasibialgebra with a quasiantipode.

If $V$ is a finite-dimensional module over a quasi-Hopf algebra $H$, then $V$ has a left dual $V^{\vee}$, defined to be its vector space dual $V^{*}$ equipped with the module structure defined by $\langle h \kappa, v\rangle=\langle\kappa, S(h) v\rangle$, and the evaluation and coevaluation defined by $\operatorname{ev}(\kappa \otimes v)=\langle\kappa, \alpha v\rangle$ and $\operatorname{db}\left(1_{k}\right)=\beta v_{i} \otimes v^{i}$, where $v_{i} \otimes v^{i} \in V \otimes V^{*}$ is the canonical element. (Here and below we use Einstein's summation convention for a repeated lower and upper index.) In particular, the category ${ }_{H} \mathcal{M}_{f}$ of finite-dimensional left $H$-modules is left and right rigid. Note that dual modules are defined in such a way that the underlying functor $\mathcal{U}:{ }_{H} \mathcal{M}_{f} \rightarrow \mathcal{M}_{k}$ preserves duals in the sense that one 
has a natural isomorphism $\mathcal{U}\left(V^{\vee}\right) \cong \mathcal{U}(V)^{*}$ (albeit incompatible with evaluation and coevaluation).

Conversely, if $H$ is a finite-dimensional quasibialgebra, the category ${ }_{H} \mathcal{M}_{f}$ is left and right rigid, and the underlying functor to $\mathcal{M}_{k}$ preserves duals in the sense that there exists a natural isomorphism $\mathcal{U}\left(V^{\vee}\right) \cong \mathcal{U}(V)^{*}$, then $H$ has a quasiantipode. See [9] for a proof, but also see [14 for an example indicating that the additional hypothesis on $\mathcal{U}$ can perhaps not be dispensed with.

We shall be needing some of the elaborate formulas for the associator and the antipode of a quasi-Hopf algebra developed in [2]. For the rest of this section, fix a quasi-Hopf algebra $H$.

First, we have an isomorphism

$$
\theta:=\theta_{A}: A \otimes H \ni a \otimes h \mapsto a \phi^{(1)} \beta S\left(h_{(1)} \phi^{(2)}\right) \otimes h_{(2)} \phi^{(3)} \in A \otimes H
$$

for all $A \in \mathcal{M}_{H}$, with inverse

$$
\theta^{-1}(a \otimes h)=a S\left(\phi^{(-1)}\right) \alpha \phi^{(-2)} h_{(1)} \otimes \phi^{(-3)} h_{(2)},
$$

slightly generalizing [2, Prop. 1.5], where it is shown that $\theta_{H}$ (in Drinfeld's notation $\varphi^{-1}$ ) is bijective with the claimed inverse. But since $\theta$ is obviously natural in $A$, the general case follows.

Further we need the twist $f$ that Drinfeld obtains to replace the fact that the antipode of an ordinary Hopf algebra is a coalgebra antimorphism. Following Drinfeld we set

$$
\begin{aligned}
\delta & :=\phi^{(-1)} \beta S\left(\phi^{(-3)}{ }_{(2)} \phi^{(3)}\right) \otimes \phi^{(-2)} \phi^{(1)} \beta S\left(\phi^{(-3)}{ }_{(1)} \phi^{(2)}\right) \\
\gamma & :=S\left(\phi^{(-1)} \phi^{(2)}\right) \alpha \phi^{(-2)} \phi^{(3)}{ }_{(1)} \otimes S\left(\phi^{(1)}\right) \alpha \phi^{(-3)} \phi^{(3)}{ }_{(2)}, \\
\mathrm{f} & :=S\left(\phi^{(-1)}{ }_{(2)}\right) \gamma^{(1)}\left(\phi^{(-2)} \beta \phi^{(-3)}\right)_{(1)} \otimes S\left(\phi^{(-1)}{ }_{(1)}\right) \gamma^{(2)}\left(\phi^{(-2)} \beta \phi^{(3)}\right)_{(2)}
\end{aligned}
$$

with the consequence that

$$
\Delta(S(h))=\mathrm{f}^{-1} \cdot(S \otimes S) \Delta^{\mathrm{cop}}(h) \cdot \mathrm{f} \quad \text { and } \quad \delta \mathrm{f}=\Delta(\beta) .
$$

The "meaning" of $\mathrm{f}$ is that it induces, for $V, W \in{ }_{H} \mathcal{M}_{f}$, the isomorphism $\mathrm{D}: W^{\vee} \otimes$ $V^{\vee} \rightarrow(V \otimes W)^{\vee}$ from (2.1); more precisely, if we identify $(V \otimes W)^{\vee}$ with $V^{*} \otimes W^{*}$ as a vector space, then $\mathrm{D}$ is the transpose of the map $\mathrm{F}: V \otimes W \rightarrow W \otimes V$ given by $\mathrm{F}(v \otimes w)=\mathrm{f}^{(2)} w \otimes \mathrm{f}^{(1)} v$.

\section{Hopf MOdulES AND THE STRUCTURE THEOREM}

Throughout this section $H$ is a quasibialgebra with associator $\phi$. If $H$ is an ordinary bialgebra, we have the well-known notion of a Hopf module, which is an important tool in Hopf algebra theory, cf. 15]. An $H$-Hopf module is by definition an $H$-module (right or left) and $H$-comodule (right or left) such that the comodule structure is a module map, or, equivalently, the module structure is a comodule map. That is, a Hopf module is an $\mathrm{H}$-module in the category of $\mathrm{H}$ comodules, or, equivalently, an $H$-comodule in the category of $H$-modules. Now in the quasibialgebra case, none of this makes sense: We do not have a notion of (ordinary) $H$-comodule, and we do not have a notion of $H$-comodule in the category of $H$-modules either, since $H$ is not a coalgebra in the category of $H$-modules. However, as Hausser and Nill [5] have observed, and used to establish integral theory for quasi-Hopf algebras along the lines of the integral theory for ordinary Hopf algebras, there is a well-behaved notion of Hopf module in ${ }_{H} \mathcal{M}_{H}^{H}$. Note first that since $H$ is a quasibialgebra, so is $H^{\mathrm{op}}$, and hence $H \otimes H^{\mathrm{op}}$, with associator 
$\phi^{(1)} \otimes \phi^{(-1)} \otimes \phi^{(2)} \otimes \phi^{(-2)} \otimes \phi^{(3)} \otimes \phi^{(-3)}$. If we identify $H \otimes H^{\mathrm{op}}$-modules with $H$-H-bimodules, this means that ${ }_{H} \mathcal{M}_{H}$ is a monoidal category with associator

$$
\begin{aligned}
\Phi:(P \otimes Q) \otimes R & \ni p \otimes q \otimes r \\
& \mapsto \phi^{(1)} p \phi^{(-1)} \otimes \phi^{(2)} q \phi^{(-2)} \otimes \phi^{(3)} r \phi^{(-3)} \in P \otimes(Q \otimes R)
\end{aligned}
$$

for $P, Q, R \in{ }_{H} \mathcal{M}_{H}$. We note that if one of $P, Q, R$ has the trivial right module structure, then $\Phi$ reduces to left multiplication by $\phi$, if one of $P, Q, R$ has the trivial left module structure, then $\Phi$ reduces to right multiplication by $\phi^{-1}$, and if one of the three has the trivial left, and another the trivial right module structure, then $\Phi$ is the identity.

Observe that $H$, with the regular $H$-bimodule structure, is a coassociative coalgebra in the monoidal category ${ }_{H} \mathcal{M}_{H}$.

Definition 3.1. A Hopf module in ${ }_{H} \mathcal{M}_{H}^{H}$ is a right $H$-comodule in the monoidal category ${ }_{H} \mathcal{M}_{H}$. A Hopf module in ${ }_{H}^{H} \mathcal{M}_{H}^{H}$ is an $H$-H-bicomodule in ${ }_{H} \mathcal{M}_{H}$.

We will freely apply the facts on comodule theory in monoidal categories to this special case. In particular, $H \in{ }_{H}^{H} \mathcal{M}_{H}^{H}$ in a natural way, and if $P \in{ }_{H} \mathcal{M}_{H}$ and $M \in{ }_{H} \mathcal{M}_{H}^{H}$, then $P \otimes M \in{ }_{H} \mathcal{M}_{H}^{H}$ with the diagonal left and right module structures, and the right comodule structure $\rho(p \otimes m)=\phi^{(-1)} p \phi^{(1)} \otimes \phi^{(-2)} m_{(0)} \phi^{(2)} \otimes$ $\phi^{(-3)} m_{(1)} \phi^{(-3)}$. We shall always use this structure without further notice. When $M \in{ }_{H} \mathcal{M}_{H}^{H}$, we denote by $M^{0}$ the $H$-bimodule $M$, with the trivial $H$-comodule structure.

Like the definition of Hopf modules, the following lemma is due to Hausser and Nill [5], at least as far as ${ }_{H} \mathcal{M}_{H}^{H}$ is concerned.

Lemma and Definition 3.2. The categories ${ }_{H} \mathcal{M}_{H}^{H}$ and ${ }_{H}^{H} \mathcal{M}_{H}^{H}$ are monoidal categories in such a way that the underlying functors to the category $\left({ }_{H} \mathcal{M}_{H}, \otimes_{H}\right)$ are strictly monoidal functors.

In particular, the tensor product of two Hopf modules $M, N$ is modelled on their tensor product over $H$. The right and, if applicable, left $H$-comodule structures on $M \otimes_{H} N$ are given by

$$
\begin{gathered}
(m \otimes n)_{(0)} \otimes(m \otimes n)_{(1)}=m_{(0)} \otimes n_{(0)} \otimes m_{(1)} n_{(1)}, \\
(m \otimes n)_{(-1)} \otimes(m \otimes n)_{(0)}=m_{(-1)} n_{(-1)} \otimes m_{(0)} \otimes n_{(0)},
\end{gathered}
$$

and the right (and left) comodule structures of $H$ are given by comultiplication.

Proof. The claimed comodule structures are trivially well-defined. To see that the right coaction $\rho$ on $M \otimes_{H} N$ is in fact a comodule structure in the bimodule category, one has to compute

$$
\begin{aligned}
(M \otimes \Delta) \rho(m \otimes n) \cdot \phi & =(m \otimes n)_{(0)} \phi^{(1)} \otimes(m \otimes n)_{(1)(1)} \phi^{(2)} \otimes(m \otimes n)_{(1)(2)} \phi^{(3)} \\
& =\left(m_{(0)} \otimes n_{(0)}\right) \phi^{(1)} \otimes\left(m_{(1)} n_{(1)}\right)_{(1)} \phi^{(2)} \otimes\left(m_{(1)} n_{(1)}\right)_{(2)} \phi^{(3)} \\
& =m_{(0)} \otimes n_{(0)} \phi^{(1)} \otimes m_{(1)(1)} n_{(1)(1)} \phi^{(2)} \otimes m_{(1)(2)} n_{(1)(2)} \phi^{(3)} \\
& =m_{(0)} \otimes \phi^{(1)} n_{(0)(0)} \otimes m_{(1)(1)} \phi^{(2)} n_{(0)(1)} \otimes m_{(1)(2)} \phi^{(3)} n_{(1)} \\
& =m_{(0)} \phi^{(1)} \otimes n_{(0)(0)} \otimes m_{(1)(1)} \phi^{(2)} n_{(0)(1)} \otimes m_{(1)(2)} \phi^{(3)} n_{(1)} \\
& =\phi^{(1)} m_{(0)(0)} \otimes n_{(0)(0)} \otimes \phi^{(2)} m_{(0)(1)} n_{(0)(1)} \otimes \phi^{(3)} m_{(1)} n_{(1)} \\
& =\phi \cdot(\rho \otimes H) \rho(m \otimes n) .
\end{aligned}
$$


The computations showing that $M \otimes_{H} N$ is, if applicable, also a left $H$-comodule, and an $H$-H-bicomodule in the bimodule category, are similar. It is trivial to verify that the coherence isomorphisms from ${ }_{H} \mathcal{M}_{H}$ are morphisms in the respective Hopf module category.

Note that the monoidal category structure of the Hopf module is not derived from general categorical reasons, but rather requires an explicit, albeit rather trivial, computation. To get it from purely categorical considerations would perhaps require a closer analysis of the interplay between the two monoidal category structures on ${ }_{H} \mathcal{M}_{H}$. On the other hand, the following monoidal category structure on ${ }_{H}^{H} \mathcal{M}_{H}^{H}$ is an entirely categorical fact:

Remark 3.3. The category ${ }_{H}^{H} \mathcal{M}_{H}^{H}$ is a monoidal category with tensor product the cotensor product of comodules within the monoidal category ${ }_{H} \mathcal{M}_{H}$.

Explicitly, this means that $M \square_{H} N \subset M \otimes N$ consists of all those elements $\sum m_{i} \otimes n_{i} \in M \otimes N$ that satisfy

$$
\sum \phi^{(1)} m_{i(0)} \otimes \phi^{(2)} m_{i(1)} \otimes \phi^{(3)} n_{i}=\sum m_{i} \phi^{(1)} \otimes n_{i(-1)} \phi^{(2)} \otimes n_{i(0)} \phi^{(3)},
$$

and the associator isomorphisms

$$
(L \underset{H}{\square} M) \underset{H}{\square} N \cong L \square_{H}(M \underset{H}{\square} N)
$$

are induced by the associator $\Phi$ in ${ }_{H} \mathcal{M}_{H}$.

Although this second monoidal category structure looks quite different from the one in Definition 3.2 it will turn out to be isomorphic provided that $H$ is a quasiHopf algebra.

We will now take the first steps towards proving Hausser and Nill's structure theorem for Hopf modules in ${ }_{H} \mathcal{M}_{H}^{H}$. We will study the functor $\mathcal{R}:{ }_{H} \mathcal{M} \rightarrow{ }_{H} \mathcal{M}_{H}^{H}$ assigning to $V \in{ }_{H} \mathcal{M}$ the cofree right $H$-comodule $V \otimes H$ over $V$ in ${ }_{H} \mathcal{M}_{H}$.

Lemma 3.4. Let $H$ be a quasibialgebra, $M \in{ }_{H} \mathcal{M}_{H}^{H}$, and $V \in{ }_{H} \mathcal{M}$. Then the canonical isomorphism $(V \otimes H) \otimes_{H} M \cong V \otimes M$ is an isomorphism in ${ }_{H} \mathcal{M}_{H}^{H}$.

Proof. The canonical isomorphism $\hat{\xi}:(V \otimes H) \otimes_{H} M \rightarrow V \otimes M$ with $\hat{\xi}^{-1}(v \otimes m)=$ $(v \otimes 1) \otimes m$ is trivially checked to be an isomorphism of $H$-bimodules. To see that it is colinear, we compute

$$
\begin{aligned}
& \rho \hat{\xi}^{-1}(v \otimes m)=\rho(v \otimes 1 \otimes m)=(v \otimes 1)_{(0)} \otimes m_{(0)} \otimes(v \otimes 1)_{(1)} m_{(1)} \\
& =\phi^{(1)} v \otimes \phi^{(2)} \otimes m_{(0)} \otimes \phi^{(3)} m_{(1)}=\phi^{(1)} v \otimes 1 \otimes \phi^{(2)} m_{(0)} \otimes \phi^{(3)} m_{(1)} \\
& =\hat{\xi}^{-1}\left(\phi^{(1)} v \otimes \phi^{(2)} m_{(0)}\right) \otimes \phi^{(3)} m_{(1)}=\left(\hat{\xi}^{-1} \otimes H\right) \rho(v \otimes m)
\end{aligned}
$$

for $v \in V$ and $m \in M$.

Definition 3.5. For $V, W \in{ }_{H} \mathcal{M}$ we define

$$
\xi:=\xi_{V W}:=\left((V \otimes H) \underset{H}{\otimes}(W \otimes H) \stackrel{\hat{\xi}}{\rightarrow} V \otimes(W \otimes H) \stackrel{\Phi^{-1}}{\longrightarrow}(V \otimes W) \otimes H\right),
$$

so that, for all $v \in V, w \in W$, and $g, h \in H$,

$$
\begin{gathered}
\xi((v \otimes g) \otimes(w \otimes h))=\phi^{(-1)} v \otimes \phi^{(-2)} g_{(1)} w \otimes \phi^{(-3)} g_{(2)} h \\
\xi^{-1}(v \otimes w \otimes h)=\left(\phi^{(1)} v \otimes 1\right) \otimes\left(\phi^{(2)} w \otimes \phi^{(3)} h\right) .
\end{gathered}
$$


Proposition 3.6. Let $H$ be a quasibialgebra. The isomorphisms $\xi$ define a monoidal functor structure on

$$
\mathcal{R}:{ }_{H} \mathcal{M} \ni V \mapsto V \otimes H \in{ }_{H} \mathcal{M}_{H}^{H}
$$

The functor $\mathcal{R}$ is fully faithful and exact.

Proof. It is trivial that $\mathcal{R}$ is faithful and exact. To see that it is full, we compute

$$
\left({ }_{H} \mathcal{M}_{H}\right)^{H}(V \otimes H, W \otimes H) \cong{ }_{H} \mathcal{M}_{H}(V \otimes H, W) \cong \operatorname{Hom}_{H-}(V, W),
$$

where the first isomorphism is the property of $W \otimes H$ of being the cofree right $H$ comodule generated by $W$ within the category ${ }_{H} \mathcal{M}_{H}$, and the second is induced by the canonical bijection $T$ : $\operatorname{Hom}_{-H}(V \otimes H, W) \cong \operatorname{Hom}(V, W)$ : The latter is given by $T(F)(v)=F(v \otimes 1)$ and $T^{-1}(f)(v \otimes h)=f(v) \varepsilon(h)$. Thus if $f=T(F)$, and $f$ is left $H$-linear, then so is $F$, since $F(g(v \otimes h))=F\left(g_{(1)} v \otimes g_{(2)} h\right)=f(g v) \varepsilon(h)=$ $g f(v) \varepsilon(h)=g F(v \otimes h)$, and conversely, if $F$ is $H$-linear, then trivially so is $f$. Note that the composed isomorphism ${ }_{H} \mathcal{M}_{H}^{H}(V \otimes H, W \otimes H) \cong \operatorname{Hom}_{H-}(V, W)$, inverse to the morphism map of the functor $\mathcal{R}$, is given by $F \mapsto(W \otimes \varepsilon) F(V \otimes \eta)$.

It remains to check that $\mathcal{R}$ is monoidal, that is, that $\xi$ is coherent. This asks for the diagrams

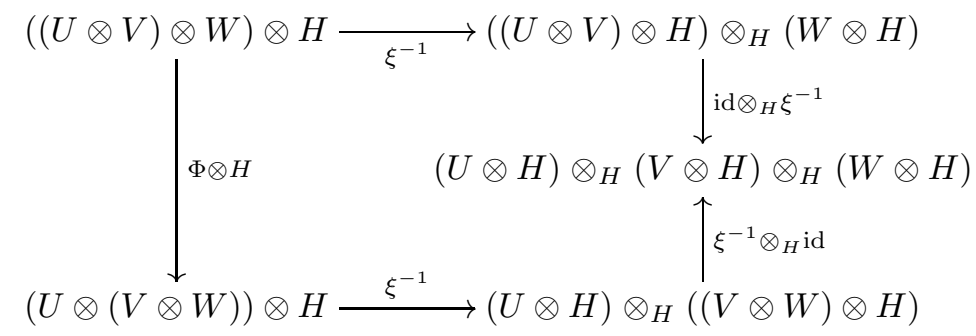

to commute for all $U, V, W \in{ }_{H} \mathcal{M}$. But upon closer inspection, this turns out to be precisely the pentagon axiom for $\Phi$ : By definition of $\xi^{-1}$, the diagrams

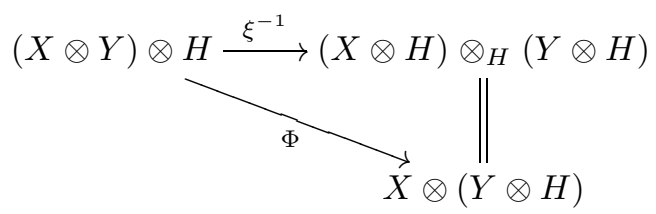

commute for all $X, Y \in{ }_{H} \mathcal{M}$. This immediately identifies all except the bottom right vertical arrow in the above pentagon with the arrows in the coherence pentagon. For that last arrow we claim that the diagram

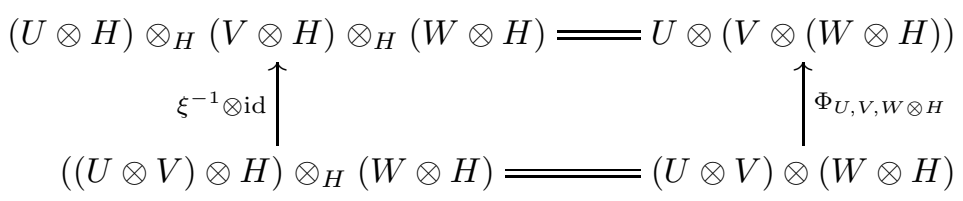


commutes as well, which can be checked by a short calculation, or by generalizing to the diagram

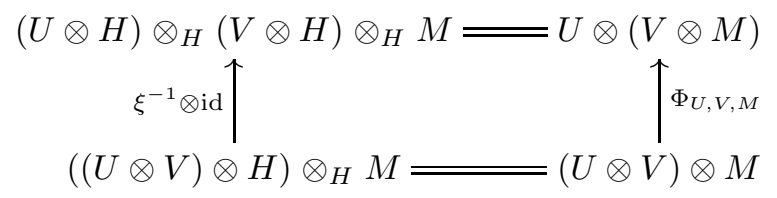

for any $M \in{ }_{H} \mathcal{M}$, which commutes since it is natural in $M$, and commutes for $M=H$.

Hausser and Nill [5] have shown that $\mathcal{R}$ is an equivalence if $H$ is a quasi-Hopf algebra (see Theorem 3.10 below), and that its quasi-inverse is monoidal, which implies that $\mathcal{R}$ is monoidal. Proving directly that $\mathcal{R}$ is monoidal, without using a quasiantipode, will allow us to draw some conclusions on the fundamental question of when a (finite-dimensional) quasibialgebra $H$ is a quasi-Hopf algebra. In [14] we have given an example of a coquasibialgebra $H$ whose finite comodule category is left and right rigid, but which is not a coquasi-Hopf algebra. In fact the dual object of a finite comodule $V$ need not have the same dimension as $V$. The next corollary shows that this rather strange phenomenon cannot occur when $H$ is finite dimensional.

Corollary 3.7. Let $H$ be a finite-dimensional quasibialgebra. Assume that the category ${ }_{H} \mathcal{M}_{f}$ of finite-dimensional left $H$-modules is rigid. Then $\operatorname{dim}\left(V^{\vee}\right)=$ $\operatorname{dim}(V)$ for all $V \in{ }_{H} \mathcal{M}_{f}$, where $V^{\vee}$ denotes the left dual of $V$ in ${ }_{H} \mathcal{M}_{f}$.

In particular, if $H$ is semisimple as an algebra, and ${ }_{H} \mathcal{M}_{f}$ is left and right rigid, then $H$ is a quasi-Hopf algebra.

Proof. Since the functor $\mathcal{R}$ from Proposition 3.6 is monoidal, so is its composition with the underlying functor ${ }_{H} \mathcal{M}_{H}^{H} \rightarrow{ }_{H} \mathcal{M}_{H}$. We use the fact that monoidal functors preserve duals. If $V \in{ }_{H} \mathcal{M}_{f}$, then the left dual of $V \otimes H$ in ${ }_{H} \mathcal{M}_{H}$ is

$$
\operatorname{Hom}_{-H}(V \otimes H, H) \cong \operatorname{Hom}(V, H) \cong V^{*} \otimes H ;
$$

so it follows that $\operatorname{dim}\left(V^{\vee} \otimes H\right)=\operatorname{dim}\left(V^{*} \otimes H\right)$, and hence $\operatorname{dim}\left(V^{\vee}\right)=\operatorname{dim}\left(V^{*}\right)=$ $\operatorname{dim}(V)$.

To conclude that $H$ is a quasi-Hopf algebra, we need to know that the underlying functor ${ }_{H} \mathcal{M}_{f} \rightarrow \mathcal{M}_{k}$ preserves duals; that is, we need an isomorphism $V^{\vee} \cong V^{*}$ of vector spaces, natural in $V \in{ }_{H} \mathcal{M}_{f}$. But if $H$ is semisimple, it suffices to fix any vector space isomorphism $V^{\vee} \cong V^{*}$ for one representative $V$ out of each isomorphism class of simple modules.

Note that we have to leave the following question open: If $H$ is a finite-dimensional quasibialgebra such that the category ${ }_{H} \mathcal{M}_{f}$ is left and right rigid, does it follow that $H$ is a quasi-Hopf algebra?

The structure theorem, Theorem 3.10, asserts that every $M \in{ }_{H} \mathcal{M}_{H}^{H}$ has the form $V \otimes H$ for some $V \in{ }_{H} \mathcal{M}$. We will now show this in the special case when $M=P \otimes H$ for some $P \in{ }_{H} \mathcal{M}_{H}$, by finding the suitable $V$ explicitly.

Definition 3.8. Let $H$ be a quasi-Hopf algebra with quasiantipode $(S, \alpha, \beta)$. We define the functor ad $(-):{ }_{H} \mathcal{M}_{H} \rightarrow{ }_{H} \mathcal{M}$ to map $P$ to ad $P:=P$ as $k$-module, with the adjoint left $H$-module structure defined by $h \cdot p:=h_{(1)} p S\left(h_{(2)}\right)$. Further we 
define, for $P \in{ }_{H} \mathcal{M}_{H}$, the map $\pi_{P}: P \otimes H \rightarrow{ }_{\text {ad }} P$ by $\pi_{P}(p \otimes h):=p \beta S(h)$, and we define

$$
\tau_{P}:=\left(P \otimes H \stackrel{P \otimes \Delta}{\longrightarrow} P \otimes\left(H^{0} \otimes H\right) \stackrel{\Phi^{-1}}{\longrightarrow}\left(P \otimes H^{0}\right) \otimes H \stackrel{\pi_{P} \otimes H}{\longrightarrow} \text { ad } P \otimes H\right)
$$

Proposition 3.9. With the notations of Definition 3.8, $\pi_{P}$ is an H-bimodule homomorphism, where ad $P$ has the trivial right $H$-module structure, and $\tau_{P}$ is an isomorphism in the category ${ }_{H} \mathcal{M}_{H}^{H}$. Both $\pi$ and $\tau$ are natural in $P \in{ }_{H} \mathcal{M}_{H}$. We have $(\mathrm{ad} P \otimes \varepsilon) \tau_{P}=\pi_{P}$.

Proof. It is quite trivial to check that $\pi$ is a bimodule map as stated, and from the way we defined $\tau$ it is evident that $\tau$ is a morphism in ${ }_{H} \mathcal{M}_{H}^{H}$. Of course $\pi$ and $\tau$ are natural, and the last equality is immediately verified. The least trivial part is bijectivity of $\tau$. Now explicitly

$$
\begin{aligned}
\tau_{P}(p \otimes h)=\phi^{(-1)} p \phi^{(1)} \beta S\left(\phi^{(-2)} h_{(1)} \phi^{(2)}\right) \otimes \phi^{(-3)} h_{(2)} \phi^{(3)} \\
=\Gamma\left(p \phi^{(1)} \beta S\left(h_{(1)} \phi^{(2)}\right) \otimes h_{(2)} \phi^{(3)}\right)=\Gamma \theta_{P}(p \otimes h),
\end{aligned}
$$

where $\theta_{P}$ uses only the right $H$-module $P$, and we have written $\Gamma(p \otimes h)=$ $\phi^{(-1)} p S\left(\phi^{(-2)}\right) \otimes \phi^{(-3)} h$. It remains to note that $\Gamma: P \otimes H \rightarrow P \otimes H$, essentially multiplication by $\phi^{-1}$, is evidently an isomorphism.

In fact we can go ahead and read off the inverse of $\tau$ :

$$
\begin{aligned}
\tau_{P}^{-1}(p \otimes h)=\theta_{P}^{-1} \Gamma^{-1}(p \otimes h)=\theta_{P}^{-1}\left(\phi^{(1)} p S\left(\phi^{(2)}\right) \otimes \phi^{(3)} h\right. \\
\quad=\phi^{(1)} p S\left(\phi^{(2)}\right) S\left(\phi^{(-1)}\right) \alpha \phi^{(-2)}\left(\phi^{(3)} h\right)_{(1)} \otimes \phi^{(-3)}\left(\phi^{(3)} h\right)_{(2)} .
\end{aligned}
$$

Theorem 3.10. Let $H$ be a quasi-Hopf algebra. Then the functor $\mathcal{R}:{ }_{H} \mathcal{M} \rightarrow$ ${ }_{H} \mathcal{M}_{H}^{H}$ from Proposition 3.6 is a category equivalence.

Proof. We have seen in Proposition 3.6 that $\mathcal{R}$ is fully faithful, so it remains to prove that $\mathcal{R}$ is essentially surjective. So let $M \in{ }_{H} \mathcal{M}_{H}^{H}$. Then $M \cong M \square_{H} H$, where the cotensor product is to be taken within the category ${ }_{H} \mathcal{M}_{H}$. In other words, we have an equalizer

$$
M \stackrel{\rho}{\longrightarrow} M^{0} \otimes H \stackrel{\Phi^{-1}(M \otimes \Delta)}{\stackrel{\rho \otimes H}{\longrightarrow}}\left(M^{0} \otimes H^{0}\right) \otimes H
$$

in ${ }_{H} \mathcal{M}_{H}^{H}$. Now by Proposition 3.9 both objects $M^{0} \otimes H$ and $\left(M^{0} \otimes H^{0}\right) \otimes H$ are in the image of $\mathcal{R}$, and since $\mathcal{R}$ is full, the parallel arrows are also in the image. Further $\mathcal{R}$ is exact, and it follows that $M$, the equalizer of the image under $\mathcal{R}$ of a pair of parallel arrows, is also in the image of $\mathcal{R}$.

As a consequence of the structure theorem, we can now show that the two monoidal category structures on ${ }_{H}^{H} \mathcal{M}_{H}^{H}$ given at the beginning of the section are essentially the same. The relevant isomorphism actually looks completely the same as that obtained for the ordinary Hopf case in [13], although the details are more involved here.

Proposition 3.11. The identity on the category ${ }_{H}^{H} \mathcal{M}_{H}^{H}$ is a monoidal functor

$$
\left({ }_{H}^{H} \mathcal{M}_{H}^{H}, \Xi\right):\left({ }_{H}^{H} \mathcal{M}_{H}^{H}, \underset{H}{\square}\right) \rightarrow\left({ }_{H}^{H} \mathcal{M}_{H}^{H}, \underset{H}{\otimes}\right)
$$


where the monoidal functor structure $\Xi$ is defined by

$$
\Xi:=\Xi_{M N}: M \underset{H}{\otimes} N \ni m \otimes n \mapsto m_{(0)} n_{(-1)} \otimes m_{(1)} n_{(0)} \in M \underset{H}{\square} N .
$$

Proof. We shall omit checking that $\Xi$ is well-defined, an $H$-bimodule map, and an $H$-bicomodule map in the bimodule category. To verify that $\Xi$ is an isomorphism, it suffices, by Theorem 3.10 to consider the case where $M=V \otimes H$ in ${ }_{H} \mathcal{M}_{H}^{H}$ for some $V \in{ }_{H} \mathcal{M}$. We have to show that the composition

$$
V \otimes N \rightarrow(V \otimes H) \underset{H}{\otimes} N \stackrel{\Xi}{\rightarrow}(V \otimes H) \underset{H}{\square} N \rightarrow V \otimes N
$$

is an isomorphism, where the first and last arrows are the canonical isomorphisms. But since $V \otimes \varepsilon: V \otimes H \rightarrow V$ is a right $H$-module map, with respect to the trivial right $H$-module structure on $V$, and since $(V \otimes \varepsilon)\left((v \otimes 1)_{(0)}\right) \otimes(v \otimes 1)_{(1)}=v \otimes 1$, this composition is simply the identity. It remains to check that $\Xi$ is coherent; that is to say, the diagrams

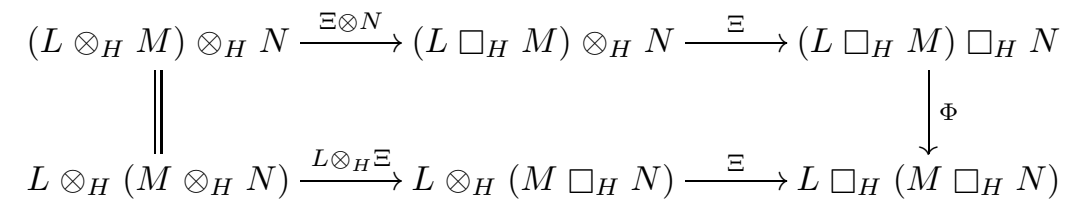

commute for all $L, M, N \in{ }_{H}^{H} \mathcal{M}_{H}^{H}$. We compute

$$
\begin{aligned}
\Phi & \Xi(\Xi \otimes N)(\ell \otimes m \otimes n) \\
& =\Phi \Xi\left(\ell_{(0)} m_{(-1)} \otimes \ell_{(1)} m_{(0)} \otimes n\right) \\
& =\Phi\left(\left(\ell_{(0)} m_{(-1)} \otimes \ell_{(1)} m_{(0)}\right)_{(0)} n_{(-1)} \otimes\left(\ell_{(0)} m_{(-1)} \otimes \ell_{(1)} m_{(0)}\right)_{(1)} n_{(0)}\right) \\
& =\Phi\left(\left(\phi^{(-1)} \ell_{(0)} m_{(-1)} \phi^{(1)} \otimes \phi^{(-2)}\left(\ell_{(1)} m_{(0)}\right)_{(0)} \phi^{(2)}\right) n_{(-1)}\right. \\
& \left.\quad \otimes \phi^{(-3)}\left(\ell_{(1)} m_{(0)}\right)_{(1)} \phi^{(3)} n_{(0)}\right) \\
= & \Phi\left(\phi^{(-1)} \ell_{(0)} m_{(-1)} \phi^{(1)} n_{(-1)(1)} \otimes \phi^{(-2)} \ell_{(1)(1)} m_{(0)(0)} \phi^{(2)} n_{(-1)(2)}\right. \\
& \left.\quad \otimes \phi^{(-3)} \ell_{(1)(2)} m_{(0)(1)} \phi^{(3)} n_{(0)}\right) \\
= & \ell_{(0)} m_{(-1)} \phi^{(1)} n_{(-1)(1)} \phi^{(-1)} \otimes \ell_{(1)(1)} m_{(0)(0)} \phi^{(2)} n_{(-1)(2)} \phi^{(-2)} \\
= & \ell_{(0)} m_{(-1)} n_{(-1)} \otimes \ell_{(1)(1)} m_{(0)(0)} n_{(0)(-1)} \otimes \ell_{(1)(2)} m_{(0)(1)} n_{(0)(0)} \\
= & \ell_{(0)} m_{(-1)} n_{(-1)} \otimes \ell_{(1)} \Xi\left(m_{(0)} \otimes n_{(0)}\right) \\
= & \Xi(\ell \otimes \Xi(m \otimes n)) . \quad \square
\end{aligned}
$$

\section{A category action}

This section is about an action of the category ${ }_{H} \mathcal{M}_{H}$ on the category ${ }_{H} \mathcal{M}$. For each $P \in{ }_{H} \mathcal{M}_{H}$ and $V \in{ }_{H} \mathcal{M}$, there is a tensor product $P \diamond V \in{ }_{H} \mathcal{M}$; in this way, the category ${ }_{H} \mathcal{M}$ is an ${ }_{H} \mathcal{M}_{H}$-category in the sense of [12], which means that there is a certain modified associativity for $\diamond$. The 'reason' for the action is the natural action of ${ }_{H} \mathcal{M}_{H}$ on ${ }_{H} \mathcal{M}_{H}^{H}$, sending $P \in{ }_{H} \mathcal{M}_{H}$ and $M \in{ }_{H} \mathcal{M}_{H}^{H}$ to $P \otimes M \in{ }_{H} \mathcal{M}_{H}^{H}$. Actually, we shall not pursue the topic of $\mathcal{C}$-categories in the present paper, but only study the relevant natural isomorphisms, and discuss the terminology at most in passing. 
Definition 4.1. Let $H$ be a quasi-Hopf algebra. For $P \in{ }_{H} \mathcal{M}_{H}$ and $V \in{ }_{H} \mathcal{M}$ we define $P \diamond V:={ }_{\text {ad }}(P \otimes V)$, and

$$
\zeta:=\zeta_{P V}:=\left(P \otimes(V \otimes H) \stackrel{\Phi^{-1}}{\longrightarrow}(P \otimes V) \otimes H \stackrel{\tau_{P \otimes V}}{\longrightarrow} \text { ad }(P \otimes V) \otimes H\right) .
$$

For $P, Q \in{ }_{H} \mathcal{M}_{H}$ and $V \in{ }_{H} \mathcal{M}$ we denote by

$$
\Omega:(P \otimes Q) \diamond V \rightarrow P \diamond(Q \diamond V)
$$

the unique $H$-module map making the diagram

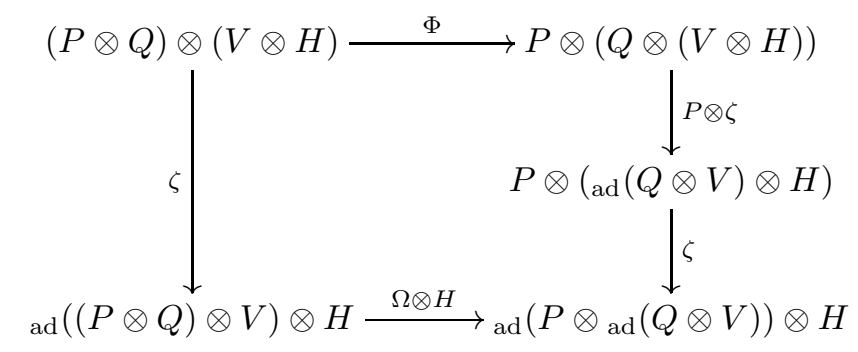

commute.

Remark 4.2. Since $\Omega:(P \otimes Q) \diamond V \rightarrow P \diamond(Q \diamond V)$ is a natural transformation, it has the form $\Omega(p \otimes q \otimes v)=\omega^{(1)} p \omega^{(5)} \otimes \omega^{(2)} q \omega^{(4)} \otimes \omega^{(3)} v$ for some element $\omega=$ $\omega^{(1)} \otimes \ldots \otimes \omega^{(5)} \in H^{\otimes 5}$.

It is easy to check that $\Omega$ is the identity whenever $P$ or $Q$ is the trivial $H$ bimodule. This implies

$$
(\varepsilon \otimes H \otimes H \otimes H \otimes \varepsilon)(\omega)=1=(H \otimes \varepsilon \otimes H \otimes \varepsilon \otimes H)(\omega)
$$

in $H \otimes H \otimes H$.

In a sense, the remark contains all we need to know about $\Omega$ when it enters below in a central rôle in the definition of Yetter-Drinfeld modules, and in the construction of the Drinfeld double. Moreover, $\zeta$ and its inverse are known explicitly, since $\tau$ and its inverse are. Thus the definition of $\Omega$ above is also by an explicit formula, and hence the element $\omega$ is, at least in principle, given explicitly. However, chasing an element around the defining diagram of $\Omega$ is far from pleasant, and thus we will be occupied for some time with deriving a manageable formula for $\omega$.

We start by investigating some special cases of the functor ad $(-)$. For $V \in{ }_{H} \mathcal{M}$ we trivially have ad $V=V$. If $A \in \mathcal{M}_{H}$, then ad $A=:{ }_{S} A$, with the left $H$-module structure defined by $h \cdot a=a S(h)$. If also $B \in \mathcal{M}_{H}$, we have an isomorphism $\mathrm{F}:{ }_{S} B \otimes{ }_{S} A \rightarrow{ }_{S}(A \otimes B)$ given by $\mathrm{F}(b \otimes a)=\tau((b \otimes a) \mathrm{f})=a \mathrm{f}^{(2)} \otimes b \mathrm{f}^{(1)}$, with the element $\mathrm{f} \in H \otimes H$ given in Section 2.3. Note further that for $V \in{ }_{H} \mathcal{M}$ and $A \in \mathcal{M}_{H}$ we have ad $(V \otimes A) \cong{ }_{\text {ad }}(A \otimes V) \cong V \otimes{ }_{S} A$ by the natural vector space isomorphisms. The map $\Psi$ in the following definition generalizes $\mathrm{F}^{-1}$.

Definition 4.3. For $P, Q \in{ }_{H} \mathcal{M}_{H}$ we define $\Psi:{ }_{\text {ad }}(P \otimes Q) \rightarrow{ }_{\text {ad }}\left(P \otimes{ }_{\text {ad }} Q\right)$ to be the unique $H$-module map making

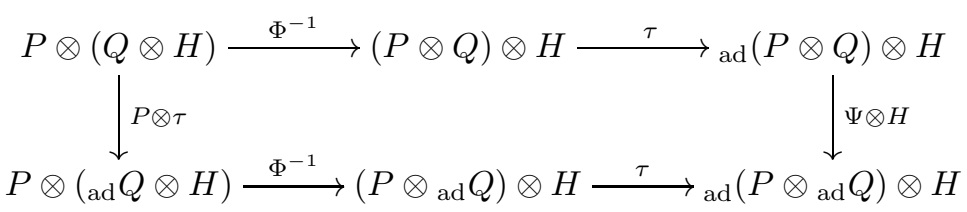

commute. 
Lemma 4.4. In the notations of Definition 4.3 we have $\Psi(p \otimes q)=\psi^{(1)} p \psi^{(4)} \otimes$ $\psi^{(2)} q \psi^{(3)}$ for all $p \in P$ and $q \in Q$, with

$$
\psi=\left(1 \otimes 1 \otimes \tau\left(\mathrm{f}^{-1}\right)\right) \cdot(H \otimes H \otimes S \otimes S)(\chi) \in H^{\otimes 4},
$$

where $\tau$ denotes the flip of tensor factors in $H \otimes H$, and

$$
\begin{aligned}
\chi & =(H \otimes \Delta \otimes H)\left(\phi^{-1}\right) \cdot\left(1 \otimes \phi^{-1}\right) \cdot(H \otimes H \otimes \Delta)(\phi) \\
& =(\phi \otimes 1) \cdot(\Delta \otimes H \otimes H)(\phi) .
\end{aligned}
$$

Proof. Forgetting Definition 4.3 for a moment, we can define a $k$-linear endomorphism $\Psi$ of $P \otimes Q$ by the formulas in the lemma. Of course $\Psi$ is natural in $P, Q \in{ }_{H} \mathcal{M}_{H}$. To prove that $\Psi:$ ad $(P \otimes Q) \rightarrow{ }_{\text {ad }}(P \otimes$ ad $Q)$ is an $H$-module map, it suffices to treat the case where $P=V \otimes A, Q=W \otimes B$ with $V, W \in{ }_{H} \mathcal{M}$ and $A, B \in \mathcal{M}_{H}$. (In fact every $P \in{ }_{H} \mathcal{M}_{H}$ is a quotient of a direct sum of copies of the $H$-bimodule $H \otimes H$; so it would be sufficient, but confusing, to assume $V=W=H$ as left and $A=B=H$ as right modules.) In this case

$$
\text { ad }(P \otimes Q) \cong(V \otimes W) \otimes{ }_{S}(A \otimes B),
$$

and

$$
{ }_{\mathrm{ad}}(P \otimes \mathrm{ad}(Q)) \cong{ }_{\mathrm{ad}}\left(P \otimes\left(W \otimes{ }_{S} B\right)\right) \cong\left(V \otimes\left(W \otimes{ }_{S} B\right)\right) \otimes_{S} A,
$$

and with these identifications, $\Psi$ is the composition of $(V \otimes W) \otimes \mathrm{F}^{-1}$ with the associativity isomorphism $(V \otimes W) \otimes\left({ }_{S} B \otimes{ }_{S} A\right) \rightarrow\left(V \otimes\left(W \otimes{ }_{S} B\right)\right) \otimes{ }_{S} A$. Now we need to check that the diagram in Definition 4.3 commutes. Since $\Psi$ is $H$-linear, all the maps in the diagram are right $H$-comodule maps in ${ }_{H} \mathcal{M}_{H}$, so it suffices to verify that the diagram commutes after composing with

$$
P \otimes Q \otimes \varepsilon:{ }_{\mathrm{ad}}(P \otimes \mathrm{ad} Q) \otimes H \rightarrow \mathrm{ad}\left(P \otimes{ }_{\mathrm{ad}} Q\right),
$$

when it becomes

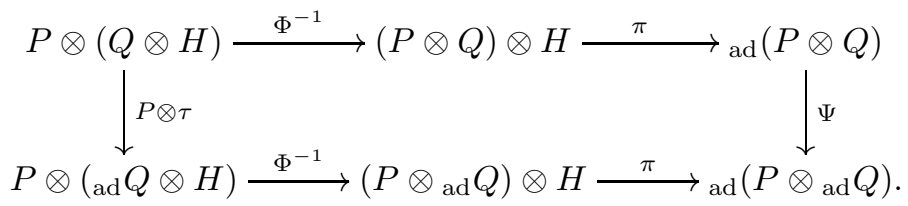

After the auxiliary calculation

$$
\begin{aligned}
\pi_{P \otimes Q} & \left(p \phi^{(1)} \otimes q \phi^{(2)} \otimes h \phi^{(3)}\right) \\
& =p \phi^{(1)} \beta_{(1)} S\left(h \phi^{(3)}\right)_{(1)} \otimes q \phi^{(2)} \beta_{(2)} S\left(h \phi^{(3)}\right)_{(2)} \\
& =p \phi^{(1)} \beta_{(1)} \mathrm{f}^{(-1)} S\left(h_{(2)} \phi^{(3)}{ }_{(2)}\right) \mathrm{f}^{(1)} \otimes q \phi^{(2)} \beta_{(2)} \mathrm{f}^{(-2)} S\left(h_{(1)} \phi^{(3)}{ }_{(1)}\right) \mathrm{f}^{(2)} \\
& =p \phi^{(1)} \delta^{(1)} S\left(h_{(2)} \phi^{(3)}{ }_{(2)}\right) \mathrm{f}^{(1)} \otimes q \phi^{(2)} \delta^{(2)} S\left(h_{(1)} \phi^{(2)}(1)\right) \mathrm{f}^{(2)} \\
& =p \beta S\left(h_{(2)} \phi^{(3)}\right) \mathrm{f}^{(1)} \otimes q \phi^{(1)} \beta S\left(h_{(1)} \phi^{(2)}\right) \mathrm{f}^{(2)}
\end{aligned}
$$

that utilizes the definition of $\delta$ and its relations to f from Section 2.3, we obtain

$$
\begin{aligned}
\Psi & \pi_{P \otimes Q} \Phi_{P Q H}^{-1}(p \otimes q \otimes h) \\
& =\Psi \pi_{P \otimes Q}\left(\phi^{(-1)} p \phi^{(1)} \otimes \phi^{(-2)} q \phi^{(2)} \otimes \phi^{(-3)} h \phi^{(3)}\right) \\
& =\Psi\left(\phi^{(-1)} p \beta S\left(\phi^{(-3)}{ }_{(2)} h_{(2)} \phi^{(3)}\right) \mathrm{f}^{(1)} \otimes \phi^{(-2)} q \phi^{(1)} \beta S\left(\phi^{(-3)}{ }_{(1)} h_{(1)} \phi^{(2)}\right) \mathrm{f}^{(2)}\right) \\
& =\chi^{(1)} \phi^{(-1)} p \beta S\left(\chi^{(4)} \phi^{(-3)}{ }_{(2)} h_{(2)} \phi^{(3)}\right) \otimes \chi^{(2)} \phi^{(-2)} q \phi^{(1)} \beta S\left(\chi^{(3)} \phi^{(-3)}{ }_{(1)} h_{(1)} \phi^{(2)}\right),
\end{aligned}
$$


which is the same as

$$
\begin{aligned}
& \pi_{P \otimes_{\mathrm{ad}} Q} \Phi_{P, \mathrm{ad}}^{-1} Q, H \\
& \quad=\pi_{P \otimes_{\mathrm{ad}} Q} \Phi_{P,{ }_{\mathrm{ad}} Q, H}^{-1}(P \otimes \tau)(p \otimes q \otimes h) \\
& \quad=\pi_{P \otimes_{\mathrm{ad}} Q}\left(\tilde{\phi}^{(-1)} p \otimes \tilde{\phi}^{(-2)}{ }_{(1)} q \phi^{(1)} \beta S\left(\tilde{\phi}^{(-2)}{ }_{(2)}^{(1)} \phi^{(-2)} h_{(1)} \phi^{(2)}\right) \otimes \tilde{\phi}^{(-3)} \phi^{(-3)} h_{(2)} \phi^{(3)}\right) \\
& \quad=\tilde{\phi}^{(-1)} p \beta S\left(\tilde{\phi}^{(-3)} \phi^{(-3)} h_{(2)} \phi^{(3)}\right) \otimes \tilde{\phi}^{(-2)}{ }_{(1)} q \phi^{(1)} \beta S\left(\tilde{\phi}_{(2)}^{(-2)} \phi^{(-2)} h_{(1)} \phi^{(2)}\right)
\end{aligned}
$$

by the definition of $\chi$.

Corollary 4.5. In the notations of Lemma 4.4 we have

$$
\omega=\left(1 \otimes 1 \otimes 1 \otimes \tau\left(\mathrm{f}^{-1}\right)\right) \cdot(H \otimes \Delta \otimes S \otimes S)(\chi) \cdot(\phi \otimes 1 \otimes 1) .
$$

Proof. Consider the diagram

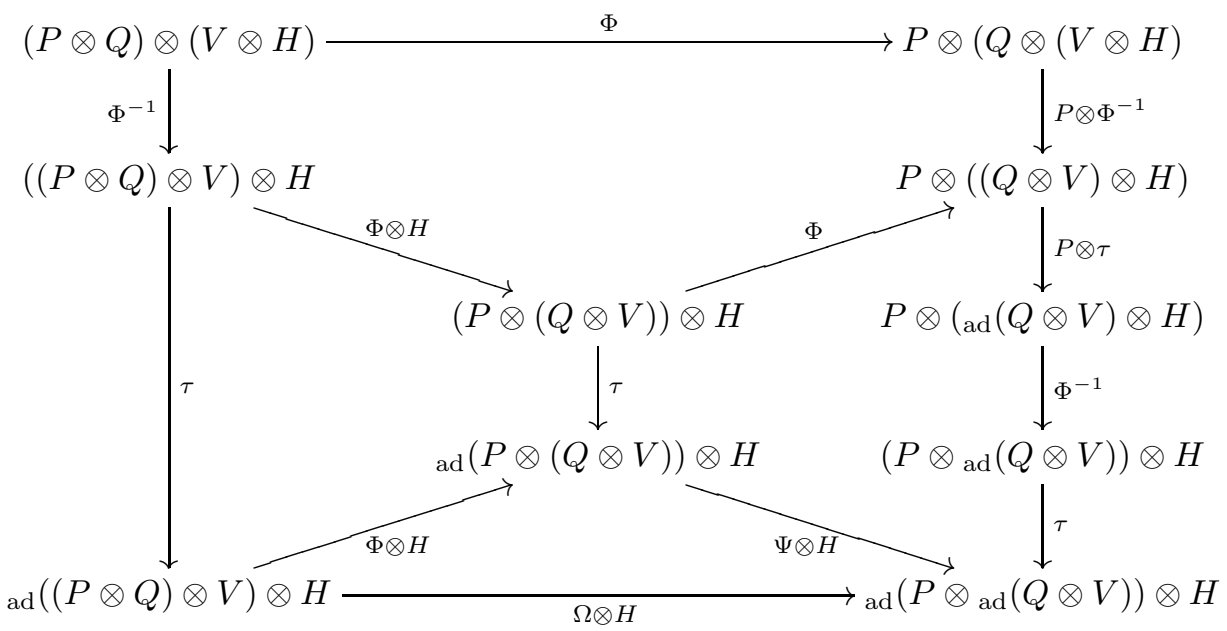

Here the pentagon on top is Mac Lane's pentagon, the quadrangle commutes by naturality of $\tau$, the hexagon is the definition of $\Psi$, and the outside commutes by the definition of $\Omega$. We conclude that $\Omega_{P Q}=\Psi_{P, Q \otimes V} \Phi_{P Q V}$.

\section{Yetter-Drinfeld MOdules OF THE SECOND KIND}

By the results of the previous section, ${ }_{H} \mathcal{M}$ is an ${ }_{H} \mathcal{M}_{H^{-}}$-category. Thus we can talk about $H$-comodules in the category ${ }_{H} \mathcal{M}$, where $H$ is a coalgebra in the category ${ }_{H} \mathcal{M}_{H}$. Since we did not elaborate on the theory of $\mathcal{C}$-categories, we give the definition explicitly:

Definition 5.1. A Yetter-Drinfeld module of the second kind is a left $H$-module equipped with an $H$-module map $\lambda^{\prime}: V \rightarrow H \diamond V$ satisfying $(\varepsilon \otimes V) \lambda^{\prime}=\mathrm{id}_{V}$, and making

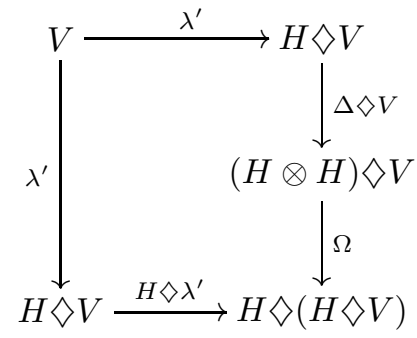


commute. We denote the category of Yetter-Drinfeld $H$-modules of the second kind by ${ }_{H}^{H} \mathcal{Y}_{2} \mathcal{D}$.

Remark 5.2. Stated differently, a Yetter-Drinfeld $H$-module of the second kind is a left $H$-module $V$ with a $k$-linear map $\lambda^{\prime}: V \rightarrow H \otimes V, \lambda^{\prime}(v)=: v_{[-1]} \otimes v_{[0]}$, satisfying

$$
\begin{gathered}
(h v)_{[-1]} \otimes(h v)_{[0]}=h_{(1)(1)} v_{[-1]} S\left(h_{(2)}\right) \otimes h_{(1)(2)} v \\
v_{[-1]} \otimes v_{[0][-1]} \otimes v_{[0][0]}=\omega^{(1)} v_{[-1](1)} \omega^{(5)} \otimes \omega^{(2)} v_{[-1](2)} \omega^{(4)} \otimes \omega^{(3)} v_{[0]},
\end{gathered}
$$

and $\varepsilon\left(v_{[-1]}\right) v_{[0]}=v$ for all $v \in V$ and $h \in H$. In particular, Yetter-Drinfeld modules of the second kind specialize to ordinary Yetter-Drinfeld modules if $H$ is an ordinary Hopf algebra.

Since objects of ${ }_{H}^{H} \mathcal{M}_{H}^{H}$ are left $H$-comodules in the ${ }_{H} \mathcal{M}_{H}$-category ${ }_{H} \mathcal{M}_{H}^{H}$, which is equivalent to the ${ }_{H} \mathcal{M}_{H}$-category ${ }_{H} \mathcal{M}$, it follows that ${ }_{H}^{H} \mathcal{M}_{H}^{H}$ is equivalent to the category ${ }_{H}^{H} \mathcal{Y}_{2} \mathcal{D}$ of left $H$-comodules in ${ }_{H} \mathcal{M}$. A more explicit statement and proof follow below:

Theorem 5.3. Let $H$ be a quasi-Hopf algebra, and $V \in{ }_{H} \mathcal{M}$. Commutativity of the diagram

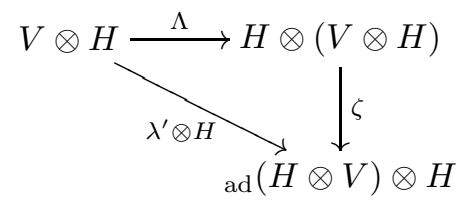

defines a bijection between maps $\Lambda$ making $V \otimes H \in{ }_{H} \mathcal{M}_{H}^{H}$ an object in ${ }_{H}^{H} \mathcal{M}_{H}^{H}$, and maps $\lambda^{\prime}$ making $V \in{ }_{H} \mathcal{M}$ a Yetter-Drinfeld module of the second kind. In particular, we have a category equivalence ${ }_{H}^{H} \mathcal{Y}_{2} \mathcal{D} \cong{ }_{H}^{H} \mathcal{M}_{H}^{H}$ induced by the equivalence ${ }_{H} \mathcal{M} \cong{ }_{H} \mathcal{M}_{H}^{H}$ from Section 圆.

Proof. Since the functor $\mathcal{R}$ is full by Proposition 3.6 we have a bijection between maps $\Lambda: V \otimes H \rightarrow H^{0} \otimes(V \otimes H)$ in ${ }_{H} \mathcal{M}_{H}^{H}$, and $H$-module maps $\lambda^{\prime}: V \rightarrow$ ad $(H \otimes V)$. We shall omit checking that $(\varepsilon \otimes V) \lambda^{\prime}=$ id is equivalent to $(\varepsilon \otimes V \otimes H) \Lambda=$ id. Then $\Lambda$ makes $V \otimes H$ an object in ${ }_{H} \mathcal{M}_{H}^{H}$ if and only if the area marked by (*) in the following diagram commutes. The diagram commutes on the outside if and only if $\lambda^{\prime}$ makes $V$ a Yetter-Drinfeld module of the second kind. Thus, if we check that all the other parts of the diagram commute, we are done. Now all three triangles are copies of (5.1), one of them tensored with $H$, and both quadrangles commute since $\zeta$ is natural. The remaining pentagon is the definition of $\Omega$. 


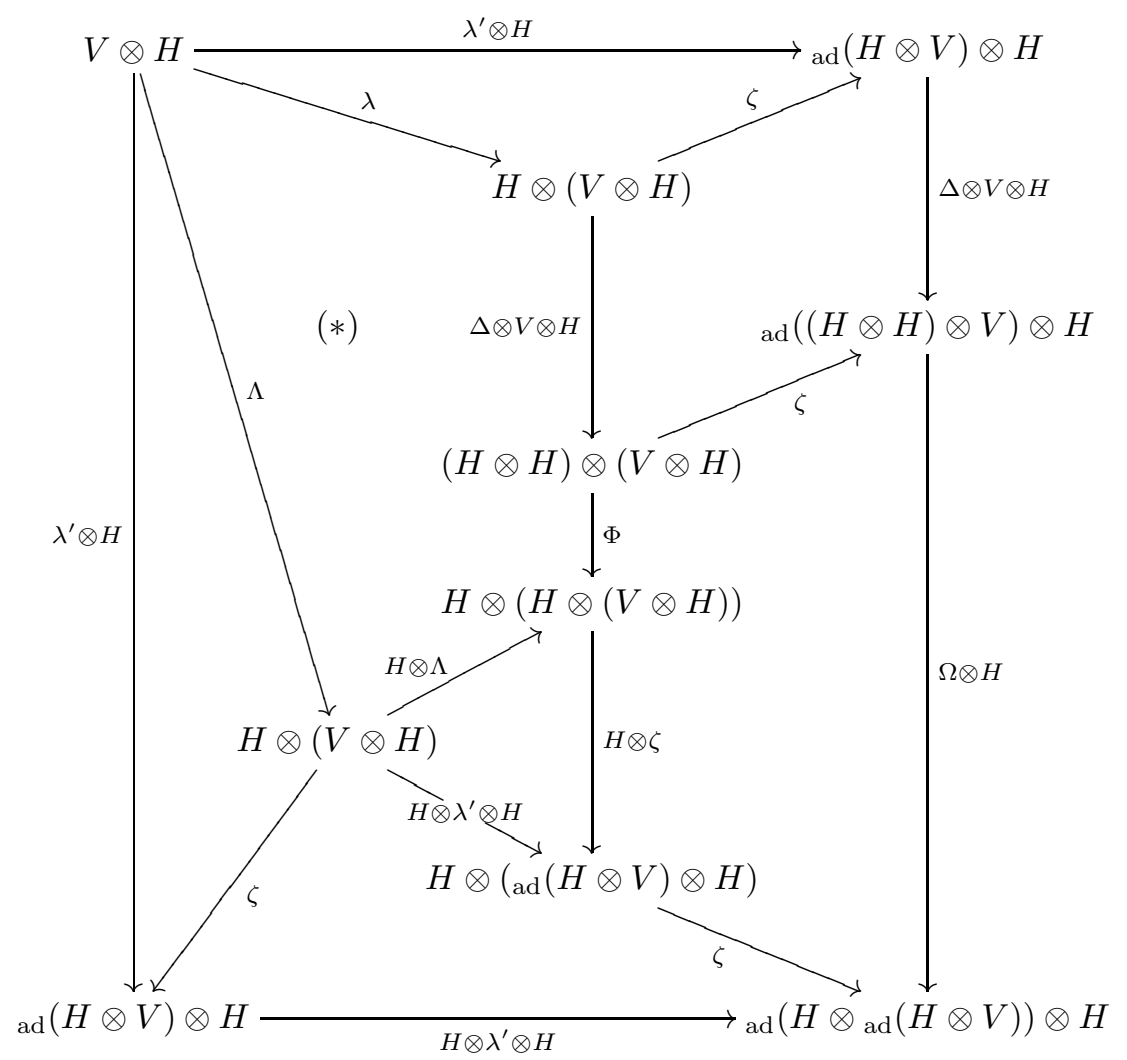

Example 5.4. For any $P \in{ }_{H}^{H} \mathcal{M}_{H}$ (the category of left $H$-comodules in ${ }_{H} \mathcal{M}_{H}$ ) we know that $P \otimes H$ is an object of ${ }_{H}^{H} \mathcal{M}_{H}^{H}$. One can check that the corresponding coaction making ad $P$ a Yetter-Drinfeld module of the second kind is given by

$$
\text { ad } P \stackrel{\Lambda_{0}}{\longrightarrow} \text { ad }(H \otimes P) \stackrel{\Psi}{\longrightarrow} \text { ad }(H \otimes \text { ad } P),
$$

when $\Lambda_{0}$ is the left $H$-comodule structure of $P$ in ${ }_{H} \mathcal{M}_{H}$.

One can apply this in particular to $P=H \in{ }_{H}^{H} \mathcal{M}_{H}$ (with the trivial right comodule structure), to get a Yetter-Drinfeld module structure on ad $H$, which is of course well-known in the ordinary Hopf algebra case.

In the following sections we shall be constructing the quantum double essentially from the situation we just established: We have a category ${ }_{H} \mathcal{M}_{H}$, which acts on the category ${ }_{H} \mathcal{M}$, and we have a coalgebra $H$ in ${ }_{H} \mathcal{M}_{H}$. As we already indicated, we will not pursue this axiomatic setup explicitly (in fact we did not even state the coherence axioms for an ${ }_{H} \mathcal{M}_{H}$-category above), nor investigate other situations to which it applies, but we hope to do so in a forthcoming paper.

\section{The Double AS AN Algebra}

Definition 6.1. Let $H$ be a finite-dimensional quasi-Hopf algebra. We endow $H^{*}$ with the (nonassociative) multiplication dual to the comultiplication of $H$. 
The Drinfeld double of $H$ is the $k$-module $D(H):=H \otimes H^{*}$ equipped with the multiplication defined by

$$
\begin{aligned}
(g \bowtie \varphi)(h & \bowtie \psi) \\
& =g h_{(1)(2)} \omega^{(3)} \bowtie\left(\omega^{(5)} \rightarrow \psi \leftarrow \omega^{(1)}\right)\left(\omega^{(4)} S\left(h_{(2)}\right) \rightarrow \varphi \leftarrow h_{(1)(1)} \omega^{(2)}\right)
\end{aligned}
$$

for $g, h \in H$ and $\varphi, \psi \in H^{*}$. Here and in the following we write $h \bowtie \psi:=h \otimes \psi \in$ $D(H)$.

Remark 6.2. By (4.1) we have $(g \bowtie \varepsilon)(h \bowtie \psi)=g h \bowtie \psi$; in particular, the embedding $\left(H \otimes \varepsilon^{*}\right): H \rightarrow D(H)$ is multiplicative, and $1 \bowtie \varepsilon$ is a left unit in $D(H)$. We also have $(1 \bowtie \varphi)(h \bowtie \varepsilon)=h_{(1)(2)} \bowtie S\left(h_{(2)}\right) \rightarrow \varphi<h_{(1)(1)}$; in particular, $1 \bowtie \varepsilon$ is a right unit.

Theorem 6.3. The Drinfeld double $D(H)$ of a finite-dimensional quasi-Hopf algebra $H$ is an associative algebra with unit $1 \otimes \varepsilon$.

An isomorphism of categories ${ }_{D(H)} \mathcal{M} \cong{ }_{H}^{H} \mathcal{Y}_{2} \mathcal{D}$ is given by assigning to a YetterDrinfeld module $V$ the left $D(H)$-module $V$ with the module structure defined by

$$
(h \bowtie \varphi) v=\left\langle\varphi, v_{[-1]}\right\rangle h v_{[0]} .
$$

Proof. Put $D:=D(H)$, and $1:=1_{D}:=1 \otimes \varepsilon \in D$. Although we do not know, as of yet, that $D$ is an associative algebra, we define the category ${ }_{D} \mathcal{M}$ of left $D$-modules to consist of $k$-modules $V$ with a map $\mu: D \otimes V \ni d \otimes v \mapsto d v \in V$ satisfying $d\left(d^{\prime} v\right)=\left(d d^{\prime}\right) v$ for all $d, d^{\prime} \in D$ and $v \in V$, as well as $1_{D} v=v$.

If $V \in{ }_{H}^{H} \mathcal{Y}_{2} \mathcal{D}$, then $V$ is in ${ }_{D} \mathcal{M}$ with the structure (6.1) by the calculation

$$
\begin{aligned}
(g & \bowtie \varphi)((h \bowtie \psi) v) \\
& =(g \bowtie \varphi)\left\langle\psi, v_{[-1]}\right\rangle h v_{[0]} \\
& =\left\langle\psi, v_{[-1]}\right\rangle\left\langle\varphi,\left(h v_{[0]}\right)_{[-1]}\right\rangle g\left(h v_{[0]}\right)_{[0]} \\
& =\left\langle\psi, v_{[-1]}\right\rangle\left\langle\varphi, h_{(1)(1)} v_{[0][-1]} S\left(h_{(2)}\right)\right\rangle g h_{(1)(2)} v_{[0][0]} \\
& =\left\langle\psi, \omega^{(1)} v_{[-1](1)} \omega^{(5)}\right\rangle\left\langle\varphi, h_{(1)(1)} \omega^{(2)} v_{[-1](2)} \omega^{(4)} S\left(h_{(2)}\right)\right\rangle g h_{(1)(2)} \omega^{(3)} v_{[0]} \\
& =\left\langle\omega^{(5)} \rightarrow \psi \leftarrow \omega^{(1)}, v_{[-1](1)}\right\rangle\left\langle\omega^{(4)} S\left(h_{(2)}\right) \rightarrow \varphi<h_{(1)(1)} \omega^{(2)}, v_{[-1](2)}\right\rangle \\
& \quad \cdot g h_{(1)(2)} \omega^{(3)} v_{[0]} \\
& =\left(g h_{(1)(2)} \omega^{(3)} \bowtie\left(\omega^{(5)} \rightarrow \psi \leftarrow \omega^{(1)}\right)\left(\omega^{(4)} S\left(h_{(2)}\right) \rightarrow \varphi \leftarrow h_{(1)(1)} \omega^{(2)}\right)\right) v
\end{aligned}
$$

If, conversely, $V \in{ }_{D} \mathcal{M}$, then $V \in{ }_{H}^{H} \mathcal{Y}_{2} \mathcal{D}$ by $h v=(h \bowtie \varepsilon) v$, and $\left\langle\varphi, v_{[-1]}\right\rangle v_{[0]}=$ $(1 \bowtie \varphi) v$, since $H \ni h \mapsto h \bowtie \varepsilon \in D$ is multiplicative and unital, and since

$$
\begin{aligned}
\left\langle\varphi,(h v)_{[-1]}\right\rangle(h v)_{[0]} & =(1 \bowtie \varphi)(h \bowtie \varepsilon) v \\
& =\left(h_{(1)(2)} \bowtie S\left(h_{(2)}\right) \rightarrow \varphi<h_{(1)(1)}\right) v \\
& =\left\langle S\left(h_{(2)}\right) \rightarrow \varphi \leftarrow h_{(1)(1)}, v_{[-1]}\right\rangle h_{(1)(2)} v_{[0]} \\
& =\left\langle\varphi, h_{(1)(1)} v_{[-1]} S\left(h_{(2)}\right)\right\rangle h_{(1)(2)} v_{[0]}
\end{aligned}
$$


and

$$
\begin{aligned}
\langle\varphi, & \left.v_{[-1]}\right\rangle\left\langle\psi, v_{[0][-1]}\right\rangle v_{[0][0]} \\
& =(1 \bowtie \psi)(1 \bowtie \varphi) v \\
& =\left(\omega^{(3)} \bowtie\left(\omega^{(5)}-\psi \leftarrow \omega^{(1)}\right)\left(\omega^{(4)}-\varphi \leftarrow \omega^{(2)}\right)\right) v \\
& =\left\langle\left(\omega^{(5)}-\psi \leftarrow \omega^{(1)}\right)\left(\omega^{(4)}-\varphi \leftarrow \omega^{(2)}\right), v_{[-1]}\right\rangle \omega^{(3)} v_{[0]} \\
& =\left\langle\omega^{(5)}-\psi \leftarrow \omega^{(1)}, v_{[-1](1)}\right\rangle\left\langle\omega^{(4)}-\varphi \leftarrow \omega^{(2)}, v_{[-1](2)}\right\rangle \omega^{(3)} v_{[0]} \\
& =\left\langle\psi, \omega^{(1)} v_{[-1](1)} \omega^{(5)}\right\rangle\left\langle\varphi, \omega^{(2)} v_{[-1](2)} \omega^{(4)}\right\rangle \omega^{(3)} v_{[0]}
\end{aligned}
$$

hold for all $v \in V, h \in H$, and $\varphi, \psi \in H^{*}$.

It remains to verify that multiplication is associative. For this it suffices to find $W \in{ }_{D} \mathcal{M}$ such that the (by definition multiplicative) map $L: D \ni d \mapsto(v \mapsto d v) \in$ $\operatorname{End}(W)$ is injective. To verify injectivity, it suffices to find $w \in \operatorname{Hom}(H, W)$ and $\vartheta \in \operatorname{Hom}(W, H)$ with $\vartheta((h \bowtie \varphi) w(g))=h\langle\varphi, g\rangle$ for all $g, h \in H$ and $\varphi \in H^{*}$, since this implies $\vartheta\left(L(h \bowtie \varphi)\left(w\left(g_{i}\right)\right)\right) \otimes g^{i}=h\left\langle\varphi, g_{i}\right\rangle \otimes g^{i}=h \otimes \varphi$ when $g_{i} \otimes g^{i} \in H \otimes H^{*}$ is the canonical element, with summation over $i$ understood.

Hence, we are looking for $W \in{ }_{H}^{H} \mathcal{Y}_{2} \mathcal{D}, w \in \operatorname{Hom}(H, W)$, and $\vartheta \in \operatorname{Hom}(W, H)$ with $\vartheta\left(\left\langle\varphi, w(g)_{[-1]}\right\rangle h w(g)_{[0]}\right)=h\langle\varphi, g\rangle$ for all $g, h \in H$ and $\varphi \in H^{*}$, which amounts to $w(g)_{[-1]} \otimes \vartheta\left(h w(g)_{[0]}\right)=g \otimes h$ for all $g, h \in H$.

Let $V$ be the left $H$-module $H$ with the trivial right $H$-module structure, and $W:={ }_{\text {ad }}(H \otimes V)$. Then $\mathcal{R}(W)={ }_{\text {ad }}(H \otimes V) \otimes H \cong(H \otimes V) \otimes H$, which is naturally an object of ${ }_{H}^{H} \mathcal{M}_{H}^{H}$ (the cofree right $H$-comodule generated by the cofree left $H$-comodule generated by $V \in{ }_{H} \mathcal{M}_{H}$ ). The corresponding map $\lambda^{\prime}$ making $W$ a Yetter-Drinfeld module of the second kind is defined to make the left hand square of the following diagram commute:

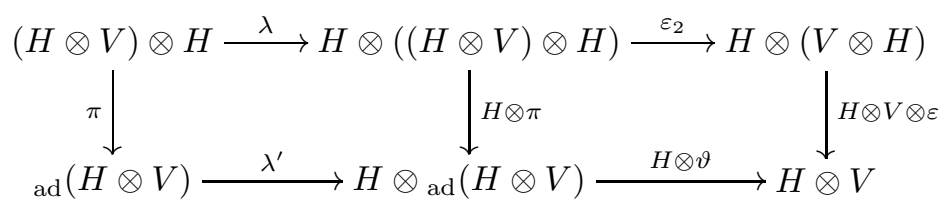

The right hand square, in which $\varepsilon_{2}$ is short for $H \otimes \varepsilon \otimes V \otimes H$, and $\vartheta:=\varepsilon \otimes$ $V:$ ad $(H \otimes V) \rightarrow V$, commutes trivially. Now the composition of the top arrows is simply $\Phi$, cf. (2.2). Hence we have

$$
(H \otimes \vartheta) \lambda^{\prime}(g \otimes v)=(H \otimes \vartheta) \lambda^{\prime} \pi(g \otimes v \otimes 1)=(H \otimes H \otimes \varepsilon) \Phi(g \otimes v \otimes 1)=g \otimes v
$$

for $g \in H$ and $v \in V$. Define $w: H \rightarrow W$ by $w(h)=h \otimes 1$. Then since $\vartheta$ is an $H$-module map, we have further $w(g)_{[-1]} \otimes \vartheta\left(h w(g)_{[0]}\right)=w(g)_{[-1]} \otimes h \vartheta\left(w(g)_{[0]}\right)=$ $g \otimes h$.

Remark 6.4. We know that the category ${ }_{H}^{H} \mathcal{Y}_{2} \mathcal{D}$ is a monoidal category, since it is equivalent to the monoidal category ${ }_{H}^{H} \mathcal{M}_{H}^{H}$. It is not hard to check that the underlying functor to $\mathcal{M}_{k}$ is a strict incoherent tensor functor. Consequently $D(H)$ is a quasibialgebra. However, it appears to be rather complicated to describe the monoidal category structure of ${ }_{H}^{H} \mathcal{Y}_{2} \mathcal{D}$, and hence the quasibialgebra structure of $D(H)$, explicitly at this point. We will attack this problem in Section 9 


\section{DuAlity AND The CENTER}

It is the author's creeping suspicion that all the material in this section is folklore. Certainly computations with 'mates' very similar to those below are well-known, like for example the formula in [7, Prop. XIV.3.1]. The use of mates to define braidings on dual vector spaces goes back at least to work of Lyubashenko [8]. However, since the precise conclusions below do not appear to be in the literature, we develop the interplay between the center construction and duality in some detail.

Definition 7.1. Let $\mathcal{C}$ be a monoidal category. The (left) weak center $\mathcal{W}(\mathcal{C})$ of $\mathcal{C}$ has as its objects pairs $\left(V, \sigma_{V,-}\right)$ in which $V$ is an object of $\mathcal{C}$, and in which $\sigma_{V, X}: V \otimes X \rightarrow X \otimes V$ is natural in $X \in \mathcal{C}$, makes

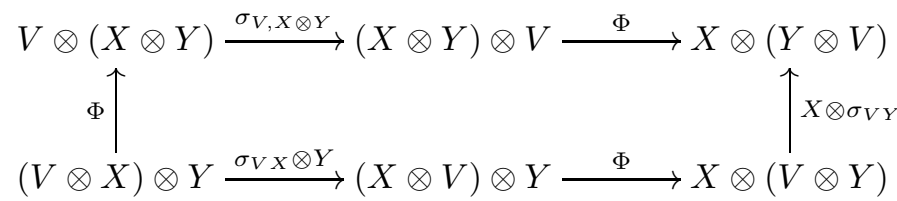

commute for all $X, Y \in \mathcal{C}$, and satisfies $\sigma_{V I}=\mathrm{id}_{V}$.

The last condition is redundant if $\sigma_{V,-}$ is an isomorphism; if this is the case we say that $\left(V, \sigma_{V,-}\right)$ is in the center $\mathcal{Z}(\mathcal{C})$.

A morphism $f:\left(V, \sigma_{V,-}\right) \rightarrow\left(W, \sigma_{W,-}\right)$ in the (weak) center is defined to be a morphism $f: V \rightarrow W$ satisfying $\sigma_{W X}(f \otimes X)=(X \otimes f) \sigma_{V X}$ for all $X \in \mathcal{C}$.

A tensor product $\left(V, \sigma_{V,-}\right) \otimes\left(W, \sigma_{W,-}\right):=\left(V \otimes W, \sigma_{V \otimes W,-}\right)$ making the (weak) center a monoidal category is defined by commutativity of

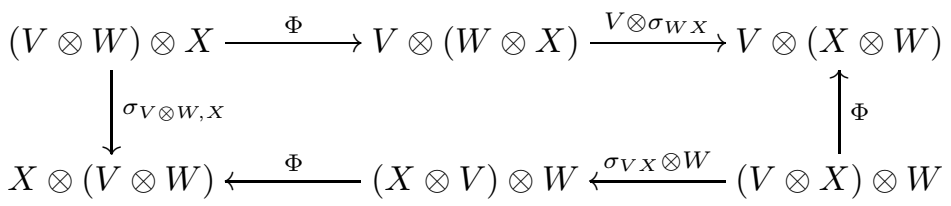

for $X \in \mathcal{C}$. The neutral object is $\left(I, \sigma_{I,-}\right)$ with $\sigma_{I X}=\mathrm{id}_{X}$.

Let $\mathcal{C}$ be a monoidal category. Recall that if $X \in \mathcal{C}$ has a left dual, then we have bijections

$$
\mathcal{C}(A \otimes X, B) \ni f \mapsto\left(f \otimes X^{\vee}\right)(A \otimes \mathrm{db}) \in \mathcal{C}\left(A, B \otimes X^{\vee}\right)
$$

with inverse $f \mapsto(B \otimes \mathrm{ev})(f \otimes X)$, and

$$
\mathcal{C}(A, X \otimes B) \ni f \mapsto(\mathrm{ev} \otimes B)\left(X^{\vee} \otimes f\right) \in \mathcal{C}\left(X^{\vee} \otimes A, B\right),
$$

with inverse $f \mapsto(X \otimes f)(\mathrm{db} \otimes A)$. (We have only given the formulas for the strict case each time.) Combining the two, we have bijections

$$
\mathcal{C}(A \otimes X, X \otimes B) \ni F \mapsto F^{b} \in \mathcal{C}\left(X^{\vee} \otimes A, B \otimes X^{\vee}\right) .
$$

Following Joyal and Street, we call $F^{b}$ the mate of $F$. We will use mates to see how the center construction behaves with respect to duals.

Lemma 7.2. Let $\mathcal{C}$ be a monoidal category, and assume that $X \in \mathcal{C}$ has a left dual $X^{\vee}$. Let $\left(V, \sigma_{V,-}\right) \in \mathcal{W}(\mathcal{C})$. Then $\sigma_{V, X^{\vee}}$ is an isomorphism. 
Proof. We assume that $\mathcal{C}$ is strict, and claim that the mate $\sigma^{b}:=\sigma_{V X}^{b}$ is inverse to $\sigma:=\sigma_{V, X^{\vee}}$. To prove that $\sigma^{b} \sigma=\mathrm{id}_{V \otimes X^{\vee}}$, it suffices to check that

$$
(V \otimes \mathrm{ev})\left(\sigma^{b} \sigma \otimes X\right)=V \otimes \mathrm{ev}: V \otimes X^{\vee} \otimes X \rightarrow V,
$$

which is done by the diagram

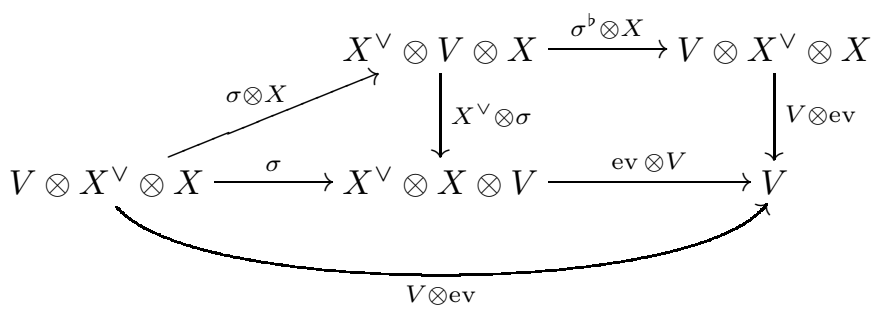

in which the curved bottom triangle is naturality of $\sigma$ applied to ev. To prove $\sigma \sigma^{b}=\mathrm{id}$, one uses a dual diagram to check that $\left(X \otimes \sigma \sigma^{\mathrm{b}}\right)(\mathrm{db} \otimes V)=(\mathrm{db} \otimes V)$.

Lemma 7.3. Let $\mathcal{C}$ be a monoidal category, and $\left(V, \sigma_{V,-}\right) \in \mathcal{Z}(\mathcal{C})$. Assume $V$ has a left dual $V^{\vee}$ in $\mathcal{C}$. Then $\left(V, \sigma_{V,-}\right)$ has a left dual in the weak center $\mathcal{W}(\mathcal{C})$.

Proof. We assume that $\mathcal{C}$ is strict, and claim that $\left(V^{\vee}, \sigma_{V^{\vee},-}\right)$, defined by $\sigma_{V^{\vee}, X}=$ $\left(\sigma_{X V}^{-1}\right)^{b}$ for $X \in \mathcal{C}$, is a left dual for $\left(V, \sigma_{V,-}\right)$ in $\mathcal{W}(\mathcal{C})$.

To show that $\left(X \otimes \sigma_{V^{\vee}, Y}\right)\left(\sigma_{V^{\vee}, X} \otimes Y\right)=\sigma_{V^{\vee}, X \otimes Y}$ for $X, Y \in \mathcal{C}$, it suffices to check that the following diagram commutes on the outside:

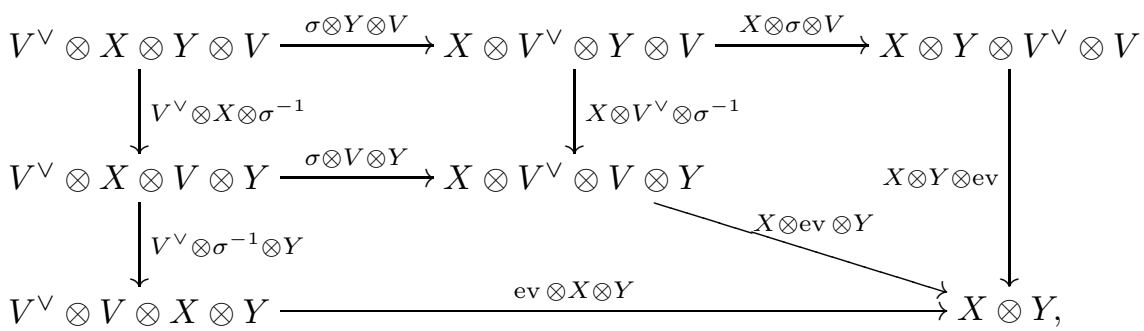

which it does since all its inner parts commute trivially or by definition of $\sigma_{V^{\vee},-}$.

Hence $\left(V^{\vee}, \sigma_{V^{\vee},-}\right)$ is in the weak center. To see that it is dual to $\left(V, \sigma_{V,-}\right)$ we have to check that ev, $\mathrm{db}$ are morphisms in the weak center. This, however, is simply the definition of $\sigma_{V^{\vee},-}$.

Corollary 7.4. Let $\mathcal{C}$ be a left and right rigid monoidal category. Then the weak center of $\mathcal{C}$ coincides with the center, and it is left and right rigid.

As a special case of the above, we also see that $\sigma_{V^{\vee}, W^{\vee}}=\sigma_{V W}^{b b}$ holds for $V \in$ $\mathcal{Z}(\mathcal{C})$, if $\mathcal{C}$ is left and right rigid. Under the usual identification $\mathrm{D}$ of $V^{\vee} \otimes W^{\vee}$ with $(W \otimes V)^{\vee}$, this amounts to saying that

$$
\sigma_{V^{\vee}, W^{\vee}}=\left(V^{\vee} \otimes W^{\vee} \stackrel{\mathrm{D}^{-1}}{\longrightarrow}(W \otimes V)^{\vee} \stackrel{\sigma_{V W}^{\vee}}{\longrightarrow}(V \otimes W)^{\vee} \stackrel{\mathrm{D}}{\longrightarrow} W^{\vee} \otimes V^{\vee}\right)
$$

\section{YeTter-DRINFELD MODULES OF THE FIRST KIND}

In this section we will deal with the version of Yetter-Drinfeld modules over quasi-Hopf algebras that was introduced (without using this term) by Majid [10] to analyze the center of the category of modules (we will find this interpretation again in Theorem 8.2). 
Definition 8.1. Let $H$ be a quasibialgebra. A Yetter-Drinfeld $H$-module of the first kind is a left $H$-module $V$ equipped with a map $\lambda: V \rightarrow H \otimes V, \lambda(v)=$ : $v_{(-1)} \otimes v_{(0)}$, satisfying $(\varepsilon \otimes V) \lambda=\mathrm{id}_{V}$,

$$
h_{(1)} v_{(-1)} \otimes h_{(2)} v_{(0)}=\left(h_{(1)} v\right)_{(-1)} h_{(2)} \otimes\left(h_{(1)} v\right)_{(0)}
$$

and

$$
\begin{aligned}
\tilde{\phi}^{(1)}\left(\phi^{(1)} v\right)_{(-1)(1)} \phi^{(2)} \otimes \tilde{\phi}^{(2)}\left(\phi^{(1)} v\right)_{(-1)(2)} \phi^{(3)} \otimes \tilde{\phi}^{(3)}\left(\phi^{(1)} v\right)_{(0)} \\
=\phi^{(1)} v_{(-1)} \otimes\left(\phi^{(2)} v_{(0)}\right)_{(-1)} \phi^{(3)} \otimes\left(\phi^{(2)} v_{(0)}\right)_{(0)}
\end{aligned}
$$

for $v \in V$ and $h \in H$. We denote by ${ }_{H}^{H} \mathcal{Y}_{1} \mathcal{D}$ the category of Yetter-Drinfeld modules of the first kind.

Theorem 8.2. Let $H$ be a quasibialgebra, and $V \in{ }_{H} \mathcal{M}$. A bijection between

(1) maps $\lambda: V \rightarrow H \otimes V, \lambda(v)=v_{(-1)} \otimes v_{(0)}$, making $V$ a Yetter-Drinfeld module of the first kind,

(2) natural transformations $\sigma_{V,-}$ making $V$ an object of the weak center,

(3) $H$-bimodule maps $\sigma: V \otimes H \rightarrow H \otimes V$ making the diagram

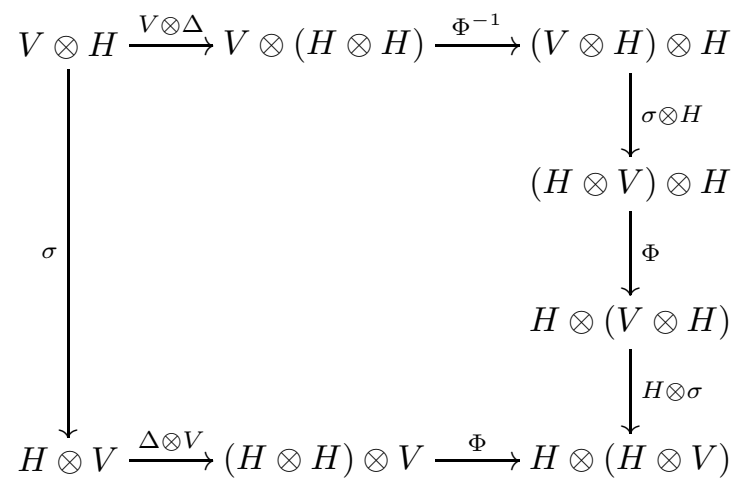

commute and satisfying $(\varepsilon \otimes V) \sigma=V \otimes \varepsilon$, and

(4) maps $\Lambda: V \otimes H \rightarrow H \otimes(V \otimes H)$ making $V \otimes H \in{ }_{H} \mathcal{M}_{H}^{H}$ an object of ${ }_{H}^{H} \mathcal{M}_{H}^{H}$, is given by the formulas $\sigma_{V X}(v \otimes x)=v_{(-1)} x \otimes v_{(0)}, \sigma=\sigma_{V H}, \lambda(v)=\sigma\left(v \otimes 1_{H}\right)$,

$$
\begin{aligned}
& \Lambda=\left(V \otimes H \stackrel{V \otimes \Delta}{\longrightarrow} V \otimes(H \otimes H) \stackrel{\Phi^{-1}}{\longrightarrow}(V \otimes H) \otimes H\right. \\
& \stackrel{\sigma \otimes H}{\longrightarrow}(H \otimes V) \otimes H \stackrel{\Phi}{\longrightarrow} H \otimes(V \otimes H)),
\end{aligned}
$$

and $\sigma=(H \otimes V \otimes \varepsilon) \Lambda$.

Proof. We get from (11) to (2) by direct computation: It is clear that $\sigma_{V X}(v \otimes x)=$ $v_{(-1)} x \otimes v_{(0)}$ defines a natural transformation, which consists of $H$-linear maps since

$$
\begin{aligned}
h \sigma_{V X}(v \otimes x)=h\left(v_{(-1)} x \otimes v_{(0)}\right) & =h_{(1)} v_{(-1)} x \otimes h_{(2)} v_{(0)} \\
& =\left(h_{(1)} v\right)_{(-1)} h_{(2)} x \otimes\left(h_{(1)} v\right)_{(0)}=\sigma_{V X}(h(v \otimes x))
\end{aligned}
$$


and satisfies

$$
\begin{aligned}
& \left(X \otimes \sigma_{V Y}\right) \Phi\left(\sigma_{V X} \otimes Y\right)(v \otimes x \otimes y) \\
& =\left(X \otimes \sigma_{V Y}\right) \Phi\left(v_{(-1)} x \otimes v_{(0)} \otimes y\right) \\
& =\left(X \otimes \sigma_{V Y}\right)\left(\phi^{(1)} v_{(-1)} x \otimes \phi^{(2)} v_{(0)} \otimes \phi^{(3)} y\right) \\
& =\phi^{(1)} v_{(-1)} x \otimes\left(\phi^{(2)} v_{(0)}\right)_{(-1)} \phi^{(3)} y \otimes\left(\phi^{(2)} v_{(0)}\right)_{(0)} \\
& =\tilde{\phi}^{(1)}\left(\phi^{(1)} v\right)_{(-1)(1)} \phi^{(2)} x \otimes \tilde{\phi}^{(2)}\left(\phi^{(1)} v\right)_{(-1)(2)} \phi^{(3)} y \otimes \tilde{\phi}^{(3)}\left(\phi^{(1)} v\right)_{(0)} \\
& =\Phi\left(\left(\phi^{(1)} v\right)_{(-1)(1)} \phi^{(2)} x \otimes\left(\phi^{(1)} v\right)_{(-1)(2)} \phi^{(3)} y \otimes\left(\phi^{(1)} v\right)_{(0)}\right) \\
& =\Phi\left(\left(\phi^{(1)} v\right)_{(-1)}\left(\phi^{(2)} x \otimes \phi^{(3)} y\right) \otimes\left(\phi^{(1)} v\right)_{(0)}\right) \\
& =\Phi \sigma_{V, X \otimes Y}\left(\phi^{(1)} v \otimes \phi^{(2)} x \otimes \phi^{(3)} y\right) \\
& =\Phi \sigma_{V, X \otimes Y} \Phi(v \otimes x \otimes y)
\end{aligned}
$$

for all $X, Y \in{ }_{H} \mathcal{M}$ and $x \in X, y \in Y$.

Given that $\left(V, \sigma_{V,-}\right)$ is in the weak center, the diagram in (3) commutes for $\sigma=\sigma_{V, H}$ : We can insert the arrow $\sigma_{V, H \otimes H}$ down the middle of the diagram; then the right hand part commutes by the defining axiom of the center, and the left hand part by naturality of $\sigma_{V,-}$ applied to $\Delta$.

The bijection between the data in (3) and (4) is essentially an observation of Tambara: In fact, for any monoidal category $\mathcal{C}$, object $V \in \mathcal{C}$, and coalgebra $H$ in $\mathcal{C}$, we have a bijection between morphisms $\sigma: V \otimes H \rightarrow H \otimes V$ in $\mathcal{C}$ satisfying the conditions in (3), and left $H$-comodule structures on $V \otimes H$ making it an $H$ - $H$ bicomodule in $\mathcal{C}$. Our case is $\mathcal{C}={ }_{H} \mathcal{M}_{H}$. Tambara [16] proves the dual statement for $\mathcal{C}=\mathcal{M}_{k}$. The general case is actually no harder, provided that $\mathcal{C}$ is strict; it is written out merely for completeness in [13. We can then apply the principle that general statements on abstract monoidal categories need only be proved in the strict case.

We go back from (3) to (11) utilizing the bijection between (3) and (41) for convenience. Any right $H$-module map $\sigma: V \otimes H \rightarrow H \otimes V$ has the form $\sigma(v \otimes h)=$ $v_{(-1)} h \otimes v_{(0)}$ for a unique map $\lambda: V \ni v \mapsto v_{(-1)} \otimes v_{(0)} \in H \otimes V$, namely $\lambda(v)=\sigma(v \otimes 1)$. Now assume that $\sigma$ fulfills the conditions in (3). Since $\sigma$ is left $H$-linear, we have $h_{(1)} v_{(-1)} \otimes h_{(2)} v_{(0)}=h \lambda(v)=h \sigma(v \otimes 1)=\sigma(h(v \otimes 1))=$ $\left(h_{(1)} v\right)_{(-1)} h_{(2)} \otimes\left(h_{(1)} v\right)_{(0)}$ for all $h \in H$ and $v \in V$. The left $H$-comodule structure on $(V \otimes H)$ is given by

$$
\begin{aligned}
\Lambda(v \otimes h) & =\Phi(\sigma \otimes H) \Phi^{-1}\left(v \otimes h_{(1)} \otimes h_{(2)}\right) \\
& =\Phi\left(\sigma\left(\phi^{(-1)} v \otimes \phi^{(-2)} h_{(1)} \otimes \phi^{(-3)} h_{(2)}\right)\right. \\
& =\Phi\left(\left(\phi^{(-1)} v\right)_{(-1)} \phi^{(-2)} h_{(1)} \otimes\left(\phi^{(-1)} v\right)_{(0)} \otimes \phi^{(-3)} h_{(2)}\right) \\
& =\phi^{(1)}\left(\phi^{(-1)} v\right)_{(-1)} \phi^{(-2)} h_{(1)} \otimes \phi^{(2)}\left(\phi^{(-1)} v\right)_{(0)} \otimes \phi^{(3)} \phi^{(-3)} h_{(2)}
\end{aligned}
$$

and hence satisfies

$$
\left(\phi^{(1)} v \otimes 1\right)_{(-1)} \phi^{(2)} \otimes\left(\phi^{(1)} v \otimes 1\right)_{(0)} \phi^{(3)}=\phi^{(1)} v_{(-1)} \otimes \phi^{(2)} v_{(0)} \otimes \phi^{(3)}
$$

and, for $\varepsilon_{2}:=(V \otimes \varepsilon): V \otimes H \rightarrow V$,

$$
(v \otimes h)_{(-1)} \otimes \varepsilon_{2}\left((v \otimes h)_{(0)}\right)=v_{(-1)} h \otimes v_{(0)} .
$$


Since $\varepsilon_{2}$ is an $H$-bimodule map, with the trivial right $H$-module structure on $V$, coassociativity for the left $H$-comodule $M=V \otimes H$ in ${ }_{H} \mathcal{M}_{H}$ implies

$$
\phi^{(1)} m_{(-1)(1)} \otimes \phi^{(2)} m_{(-1)(2)} \otimes \phi^{(3)} \varepsilon_{2}\left(m_{(0)}\right)=m_{(-1)} \otimes m_{(0)(-1)} \otimes \varepsilon_{2}\left(m_{(0)(0)}\right),
$$

so that finally

$$
\begin{aligned}
\phi^{(1)} v_{(-1)} \otimes & \left(\phi^{(2)} v_{(0)}\right)_{(-1)} \phi^{(3)} \otimes\left(\phi^{(2)} v_{(0)}\right)_{(0)} \\
= & \phi^{(1)} v_{(-1)} \otimes\left(\phi^{(2)} v_{(0)} \otimes \phi^{(3)}\right)_{(-1)} \otimes \varepsilon_{2}\left(\left(\phi^{(2)} v_{(0)} \otimes \phi^{(3)}\right)_{(0)}\right) \\
= & \left(\phi^{(1)} v \otimes 1\right)_{(-1)} \phi^{(2)} \otimes\left(\left(\phi^{(1)} v \otimes 1\right)_{(0)} \phi^{(3)}\right)_{(-1)} \\
& \otimes \varepsilon_{2}\left(\left(\left(\phi^{(1)} v \otimes 1\right)_{(0)} \phi^{(3)}\right)_{(0)}\right) \\
= & \left(\phi^{(1)} v \otimes 1\right)_{(-1)} \phi^{(2)} \otimes\left(\phi^{(1)} v \otimes 1\right)_{(0)(-1)} \phi^{(3)} \otimes \varepsilon_{2}\left(\left(\phi^{(1)} v \otimes 1\right)_{(0)(0)}\right) \\
= & \tilde{\phi}^{(1)}\left(\phi^{(1)} v \otimes 1\right)_{(-1)(1)} \phi^{(2)} \otimes \tilde{\phi}^{(2)}\left(\phi^{(1)} v \otimes 1\right)_{(-1)(2)} \phi^{(3)} \\
& \otimes \varepsilon_{2}\left(\tilde{\phi}^{(3)}\left(\phi^{(1)} v \otimes 1\right)_{(0)}\right) \\
= & \tilde{\phi}^{(1)}\left(\phi^{(1)} v\right)_{(-1)(1)} \phi^{(2)} \otimes \tilde{\phi}^{(2)}\left(\phi^{(1)} v\right)_{(-1)(2)} \phi^{(3)} \otimes \tilde{\phi}^{(3)}\left(\phi^{(1)} v\right)_{(0)} .
\end{aligned}
$$

Corollary 8.3. Let $H$ be a quasi-Hopf algebra. Then we have category equivalences

$$
{ }_{H}^{H} \mathcal{Y}_{2} \mathcal{D} \cong{ }_{H}^{H} \mathcal{M}_{H}^{H} \cong{ }_{H}^{H} \mathcal{Y} \mathcal{D} \cong \mathcal{W}\left({ }_{H} \mathcal{M}\right) \cong \mathcal{Z}\left({ }_{H} \mathcal{M}\right),
$$

which are monoidal category equivalences with a suitably defined monoidal category structure on ${ }_{H}^{H} \mathcal{Y}_{1} \mathcal{D}$.

We shall spend some time now finding out how these category equivalences look explicitly. The equivalence ${ }_{H}^{H} \mathcal{Y}_{1} \mathcal{D} \cong \mathcal{W}\left({ }_{H} \mathcal{M}\right)$ was of course given in Theorem 8.2 The resulting monoidal category structure on ${ }_{H}^{H} \mathcal{Y}_{1} \mathcal{D}$ is given by endowing the tensor product $H$-module of $V, W \in{ }_{H}^{H} \mathcal{Y}_{1} \mathcal{D}$ with the 'comodule' structure

$$
\lambda:=\left(V \otimes W \stackrel{V \otimes W \otimes \eta}{\longrightarrow}(V \otimes W) \otimes H \stackrel{\sigma_{V \otimes W, H}}{\longrightarrow} H \otimes(V \otimes W)\right),
$$

that is,

$$
\begin{aligned}
\lambda(v \otimes w) & =\sigma_{V \otimes W, H}(v \otimes w \otimes 1) \\
& =\Phi\left(\sigma_{V H} \otimes W\right) \Phi^{-1}\left(V \otimes \sigma_{W H}\right) \Phi(v \otimes w \otimes 1) \\
& =\Phi\left(\sigma_{V H} \otimes W\right) \Phi^{-1}\left(\phi^{(1)} v \otimes\left(\phi^{(2)} w\right)_{(-1)} \otimes\left(\phi^{(3)} w\right)_{(0)}\right) \\
& =\Phi\left(\sigma_{V H} \otimes W\right)\left(\phi^{(-1)} \phi^{(1)} v \otimes \phi^{(-2)}\left(\phi^{(2)} w\right)_{(-1)} \otimes \phi^{(-3)}\left(\phi^{(3)} w\right)_{(0)}\right) \\
= & \tilde{\phi}^{(1)}\left(\phi^{(-1)} \phi^{(1)} v\right)_{(-1)} \phi^{(-2)}\left(\phi^{(2)} w\right)_{(-1)} \\
& \otimes \tilde{\phi}^{(2)}\left(\phi^{(-1)} \phi^{(1)} v\right)_{(0)} \otimes \tilde{\phi}^{(3)} \phi^{(-3)}\left(\phi^{(3)} w\right)_{(0)}
\end{aligned}
$$

The equivalence ${ }_{H}^{H} \mathcal{M}_{H}^{H} \cong{ }_{H}^{H} \mathcal{Y}_{1} \mathcal{D}$ is also in Theorem 8.2 while the equivalence ${ }_{H}^{H} \mathcal{M}_{H}^{H} \cong{ }_{H}^{H} \mathcal{Y}_{2} \mathcal{D}$ is described in Theorem 5.3 We are interested in computing the composition ${ }_{H}^{H} \mathcal{Y}_{1} \mathcal{D} \cong{ }_{H}^{H} \mathcal{Y}_{2} \mathcal{D}$. This composition leaves the underlying $H$-modules fixed and only translates between the two 'comodule' structures $\lambda$ for $V \in{ }_{H}^{H} \mathcal{Y}_{1} \mathcal{D}$ and $\lambda^{\prime}$ for $V \in{ }_{H}^{H} \mathcal{Y}_{2} \mathcal{D}$ as follows: Given $\lambda$, we have

$$
\lambda^{\prime}(v)=(H \otimes V \otimes \varepsilon) \zeta \Lambda(v \otimes 1)=\left(\phi^{(-1)} v\right)_{(-1)} \phi^{(-2)} \beta S\left(\phi^{(-3)}\right) \otimes\left(\phi^{(-1)} v\right)_{(0)},
$$


and given $\lambda^{\prime}$, we have

$$
\begin{aligned}
\lambda(v) & =(H \otimes V \otimes \varepsilon) \Lambda(v \otimes 1) \\
& =(H \otimes V \otimes \varepsilon) \zeta^{-1}\left(v_{[-1]} \otimes v_{[0]} \otimes 1\right) \\
& =(H \otimes V \otimes \varepsilon) \Phi^{-1} \tau^{-1}\left(v_{[-1]} \otimes v_{[0]} \otimes 1\right) \\
& =(H \otimes V \otimes \varepsilon) \tau^{-1}\left(v_{[-1]} \otimes v_{[0]} \otimes 1\right) \\
& =\phi_{(1)}^{(1)} v_{[-1]} S\left(\phi^{(2)}\right) \alpha \phi^{(3)} \otimes \phi_{(2)}^{(1)} v_{[0]}
\end{aligned}
$$

9. The Double AS A QUASITRIANgular QUASI-HopF AlgEBRA

We now use Yetter-Drinfeld modules of the first kind to describe the quasitriangular quasi-Hopf algebra structure of the Drinfeld double. First, we need to find the counterpart in $D(H)$ for the equivalence ${ }_{H}^{H} \mathcal{Y}_{1} \mathcal{D} \cong{ }_{H}^{H} \mathcal{Y}_{2} \mathcal{D}$.

Definition 9.1. Let $H$ be a finite-dimensional quasi-Hopf algebra. We define a $\operatorname{map} \mathbf{T}: H^{*} \rightarrow D(H)$ by

$$
\mathbf{T}(\varphi)=\phi_{(2)}^{(1)} \bowtie S\left(\phi^{(2)}\right) \alpha \phi^{(3)} \rightarrow \varphi<\phi^{(1)}{ }_{(1)} .
$$

Lemma 9.2. Let $H$ be a finite-dimensional quasi-Hopf algebra. Then $D(H)$ is generated as an algebra by $H$ and the image of $\mathbf{T}$. The equivalence ${ }_{D(H)} \mathcal{M} \cong{ }_{H}^{H} \mathcal{Y}_{1} \mathcal{D}$ is described by the formula $\mathbf{T}(\varphi) v=\left\langle\varphi, v_{(-1)}\right\rangle v_{(0)}$ for all $v \in V \in{ }_{H}^{H} \mathcal{Y}_{1} \mathcal{D}$.

Proof. For $v \in V \in{ }_{H}^{H} \mathcal{Y}_{1} \mathcal{D} \cong{ }_{D(H)} \mathcal{M}$ we have

$$
\begin{aligned}
\mathbf{T}(\varphi) v=\left\langle S\left(\phi^{(2)}\right) \alpha \phi^{(3)}\right. & \left.\rightarrow \varphi \leftarrow \phi^{(1)}{ }_{(1)}, v_{[-1]}\right\rangle \phi^{(1)}{ }_{(2)} v_{[0]} \\
& =\left\langle\varphi, \phi^{(1)}{ }_{(1)} v_{[-1]} S\left(\phi^{(2)}\right) \alpha \phi^{(3)}\right\rangle \phi^{(1)}{ }_{(2)} v_{[0]}=\left\langle\varphi, v_{(-1)}\right\rangle v_{(0)}
\end{aligned}
$$

On the other hand,

$$
\begin{aligned}
(1 \bowtie \varphi) v=\left\langle\varphi, v_{[-1]}\right\rangle v_{[0]}=\left\langle\varphi,\left(\phi^{(1)} v\right)_{(-1)} \phi^{(-2)} \beta S\left(\phi^{(-3)}\right)\right\rangle\left(\phi^{(-1)} v\right)_{(0)} & \\
= & \mathbf{T}\left(\phi^{(-2)} \beta S\left(\phi^{(-3)}\right) \rightarrow \varphi\right) \phi^{(-1)} v
\end{aligned}
$$

for all $v$ implies $1 \bowtie \varphi=\mathbf{T}\left(\phi^{(-2)} \beta S\left(\phi^{(-3)}\right) \rightarrow \varphi\right) \phi^{(-1)}$, so that in particular $D(H)$ is generated as an algebra by $H \subset D(H)$ and $\mathbf{T}\left(H^{*}\right)$.

Theorem 9.3. The Drinfeld double of a finite-dimensional quasi-Hopf algebra $H$ has a unique quasibialgebra structure such that $H \subset D(H)$ is a subquasibialgebra, and

$$
\Delta(\mathbf{T}(\varphi))=\tilde{\phi}^{(2)} \mathbf{T}\left(\varphi_{(1)}<\tilde{\phi}^{(1)}\right) \phi^{(-1)} \phi^{(1)} \otimes \tilde{\phi}^{(3)} \phi^{(-3)} \mathbf{T}\left(\varphi_{(2)}\left\llcorner\phi^{(-2)}\right) \phi^{(3)}\right.
$$

for all $\varphi \in H^{*} . D(H)$ has a unique quasiantipode such that the inclusion $H \subset D(H)$ preserves quasiantipodes, and

$$
S(\mathbf{T}(\varphi))=\mathrm{f}^{(2)} \mathbf{T}\left(\mathrm{f}^{(-2)} \longrightarrow S^{-1}(\varphi)<\mathrm{f}^{(1)}\right) \mathrm{f}^{(-1)}
$$

for all $\varphi \in H^{*}$, where we have used the same symbol $S$ to denote the dual automorphism of $H^{*}$. Moreover, $D(H)$ is quasitriangular with $R$-matrix

$$
\mathcal{R}=\mathbf{T}\left(h^{i}\right) \otimes\left(h_{i} \bowtie \varepsilon\right),
$$

where $h_{i} \otimes h^{i} \in H \otimes H^{*}$ is the canonical element, with summation over $i$ understood. 
Proof. It is clear that $D(H)$ has a quasibialgebra structure, with $H$ as a subquasibialgebra, since ${ }_{D(H)} \mathcal{M}_{f}$ is a monoidal category by Corollary [8.3, and the underlying functor to ${ }_{H} \mathcal{M}_{f}$ is monoidal. Now for $V, W \in{ }_{D(H)} \mathcal{M} \cong{ }_{H}^{H} \mathcal{Y}_{1} \mathcal{D}$ and elements $v \in V, w \in W$ the calculation

$$
\begin{aligned}
\mathbf{T}(\varphi)(v \otimes w)= & (\varphi \otimes V \otimes W) \lambda(v \otimes w) \\
=\left\langle\varphi, \tilde{\phi}^{(1)}\left(\phi^{(-1)} \phi^{(1)} v\right)_{(-1)} \phi^{(-2)}\left(\phi^{(2)} w\right)_{(-1)}\right\rangle & \quad \tilde{\phi}^{(2)}\left(\phi^{(-1)} \phi^{(1)} v\right)_{(0)} \otimes \tilde{\phi}^{(3)} \phi^{(-3)}\left(\phi^{(3)} w\right)_{(0)} \\
=\left\langle\varphi_{(1)},\right. & \left.\tilde{\phi}^{(1)}\left(\phi^{(-1)} \phi^{(1)} v\right)_{(-1)}\right\rangle\left\langle\varphi_{(2)}, \phi^{(-2)}\left(\phi^{(2)} w\right)_{(-1)}\right\rangle \\
& \cdot \tilde{\phi}^{(2)}\left(\phi^{(-1)} \phi^{(1)} v\right)_{(0)} \otimes \tilde{\phi}^{(3)} \phi^{(-3)}\left(\phi^{(3)} w\right)_{(0)} \\
=\left\langle\varphi_{(1)}<\right. & \left.\left.\tilde{\phi}^{(1)},\left(\phi^{(-1)} \phi^{(1)} v\right)_{(-1)}\right\rangle\left\langle\varphi_{(2)} \leftarrow \phi^{(-2)}, \phi^{(2)} w\right)_{(-1)}\right\rangle \\
& \cdot \tilde{\phi}^{(2)}\left(\phi^{(-1)} \phi^{(1)} v\right)_{(0)} \otimes \tilde{\phi}^{(3)} \phi^{(-3)}\left(\phi^{(3)} w\right)_{(0)} \\
= & \tilde{\phi}^{(2)} \mathbf{T}\left(\varphi_{(1)} \leftarrow \tilde{\phi}^{(1)}\right) \phi^{(-1)} \phi^{(1)} v \otimes \tilde{\phi}^{(3)} \phi^{(-3)} \mathbf{T}\left(\varphi_{(2)} \leftarrow \phi^{(-2)}\right) \phi^{(3)} w
\end{aligned}
$$

proves (9.1) (uniqueness is obvious since $H$ and the image of $\mathbf{T}$ generate the algebra $D(H))$.

Since the center of ${ }_{H} \mathcal{M}_{f}$ is left and right rigid, with dual objects and evaluation and coevaluation maps the same as in ${ }_{H} \mathcal{M}_{f}$, it is clear that $D(H)$ has a quasiantipode $(\hat{S}, \hat{\alpha}, \hat{\beta}$ ) with $\hat{\alpha}=\alpha, \hat{\beta}=\beta$, and $\hat{S}(h)=S(h)$ for $h \in H$ (which is what we mean by the inclusion $H \subset D(H)$ preserving quasiantipodes). To determine the quasiantipode explicitly, we use (7.1) to compute, for $V \in{ }_{D(H)} \mathcal{M}_{f}$ and $W \in{ }_{H} \mathcal{M}_{f}, v \in V, \nu \in V^{*}, w \in W$, and $\kappa \in W^{*}:$

$$
\begin{aligned}
\left\langle\nu_{(-1)} \kappa \otimes \nu_{(0)}, w \otimes v\right\rangle & =\left\langle\sigma_{V^{\vee}, W^{\vee}}(\nu \otimes \kappa), w \otimes v\right\rangle \\
& =\left\langle\mathrm{D}^{-1} \sigma^{*} \mathrm{D}(\nu \otimes \kappa), w \otimes v\right\rangle \\
& =\left\langle\sigma^{*} \mathrm{D}(\nu \otimes \kappa), \mathrm{f}^{(-1)} v \otimes \mathrm{f}^{(-2)} w\right\rangle \\
& =\left\langle\mathrm{D}(\nu \otimes \kappa),\left(\mathrm{f}^{(-1)} v\right)_{(-1)} f^{(-2)} w \otimes\left(\mathrm{f}^{(-1)} v\right)_{(0)}\right\rangle \\
& =\left\langle\nu \otimes \kappa, \mathrm{f}^{(2)}\left(\mathrm{f}^{(-1)} v\right)_{(0)} \otimes \mathrm{f}^{(1)}\left(\mathrm{f}^{(-1)} v\right)_{(-1)} \mathrm{f}^{(-2)} w\right\rangle \\
& =\left\langle\nu, \mathrm{f}^{(2)}\left(\mathrm{f}^{(-1)} v\right)_{(0)}\right\rangle\left\langle S^{-1}\left(\mathrm{f}^{(1)}\left(\mathrm{f}^{(-1)} v\right)_{(-1)} \mathrm{f}^{(-2)}\right) \kappa\right\rangle,
\end{aligned}
$$

which implies

$$
\nu_{(-1)}\left\langle\nu_{(0)}, v\right\rangle=\left\langle\nu, \mathrm{f}^{(2)}\left(\mathrm{f}^{(-1)} v\right)_{(0)}\right\rangle S^{-1}\left(\mathrm{f}^{(1)}\left(\mathrm{f}^{(-1)} v\right)_{(-1)} \mathrm{f}^{(-2)}\right) \in H,
$$

and hence, for $\varphi \in H^{*}$,

$$
\begin{aligned}
\langle\mathbf{T}(\varphi) \nu, v\rangle & =\left\langle\varphi, \nu_{(-1)}\right\rangle\left\langle\nu_{(0)}, v\right\rangle \\
& =\left\langle\nu, \mathrm{f}^{(2)}\left(\mathrm{f}^{(-1)} v\right)_{(0)}\right\rangle\left\langle S^{-1}(\varphi), \mathrm{f}^{(1)}\left(\mathrm{f}^{(-1)} v\right)_{(-1)} \mathrm{f}^{(-2)}\right\rangle \\
& =\left\langle\nu, \mathrm{f}^{(2)} \mathbf{T}\left(\mathrm{f}^{(-2)}-S^{-1}(\varphi)-\mathrm{f}^{(1)}\right) \mathrm{f}^{(-1)} v\right\rangle .
\end{aligned}
$$

This proves (9.2), which clearly determines $S$ uniquely.

Finally, without recalling the axiomatics of quasitriangular quasibialgebras, we note that $D(H)$ has a quasitriangular structure since ${ }_{D(H)} \mathcal{M} \cong \mathcal{Z}\left({ }_{H} \mathcal{M}\right)$ is braided, and to see that the element $\mathcal{R}$ defined by (9.3) is the universal $R$-matrix, we need 
only calculate

$$
\begin{aligned}
\mathcal{R}^{(2)} w \otimes \mathcal{R}^{(1)} v=\left(h_{i} \bowtie \varepsilon\right) w \otimes \mathbf{T}\left(h^{i}\right) v \\
\quad=h_{i} w \otimes\left\langle h^{i}, v_{(-1)}\right\rangle v_{(0)}=v_{(-1)} w \otimes v_{(0)}=\sigma_{V W}(v \otimes w)
\end{aligned}
$$

for $v \in V \in{ }_{D(H)} \mathcal{M}$ and $w \in W \in{ }_{H} \mathcal{M}$.

Remark 9.4. Using Lemma 9.2, the definition of a Yetter-Drinfeld module of the first kind yields the relations

$$
\begin{gathered}
h_{(2)} \mathbf{T}\left(\varphi<h_{(1)}\right)=\mathbf{T}\left(h_{(2)} \rightarrow \varphi\right) h_{(1)}, \\
\mathbf{T}\left(\phi^{(3)} \rightarrow \psi\right) \mathbf{T}\left(\varphi<\phi^{(1)}\right) \phi^{(3)}=\tilde{\phi}^{(3)} \mathbf{T}\left(\left(\phi^{(2)} \rightarrow \varphi<\tilde{\phi}^{(1)}\right)\left(\phi^{(3)} \rightarrow \psi<\tilde{\phi}^{(2)}\right)\right) \phi^{(1)}
\end{gathered}
$$

in $D(H)$, for $h \in H$ and $\varphi, \psi \in H^{*}$, which appear to be defining relations for $D(H)$ if we throw in the relations expressing that $H \hookrightarrow D(H)$ is an algebra map. Using the explicit expression for $\varphi \otimes h=\theta_{H^{*}}^{-1} \theta_{H^{*}}(\varphi \otimes h)$, one can rewrite the first relation as

$$
h \mathbf{T}(\varphi)=\phi^{(-3)} \mathbf{T}\left(\left(h_{(2)} \phi^{(3)}\right)_{(2)} \rightarrow \varphi<\phi^{(1)} \beta S\left(\phi^{(-1)} h_{(1)} \phi^{(2)}\right) \alpha \phi^{(-2)}\right)\left(h_{(2)} \phi^{(3)}\right)_{(1)},
$$

which can, by and large, be understood as a commutation relation shifting $h$ from the left to the right of the image of $\mathbf{T}$. On the grounds of similar formulas, Majid claims (with slight differences in conventions) in [10] that it is clear that $H \otimes H^{*}$ has an algebra structure such that an equivalence ${ }_{H}^{H} \mathcal{Y}_{1} \mathcal{D} \cong{ }_{H^{*} \otimes H} \mathcal{M}$ can be described by $(h \otimes \varphi) v=\left\langle\varphi,(h v)_{(-1)}\right\rangle(h v)_{(0)}$. However, the obstinate appearance of the 'element' $\phi^{(-3)} \in H$ to the left of $\mathbf{T}(-)$ in the above formula for $h \mathbf{T}(\varphi)$ makes it quite unclear how to use this formula to define a multiplication in $H^{*} \otimes H$ so that $\varphi \otimes h$ would correspond to $\mathbf{T}(\varphi) h$ in our $D(H)$. And even if such a multiplication were given in 10], it would remain to check that it is associative, which is not guaranteed by the mere fact that 'modules' over $H^{*} \otimes H$ classify objects of the center, as long as we do not know any objects in the center explicitly.

It appears that the idea of Hausser and Nill to use different generators for $D(H)$ to describe its multiplication, and its quasi-Hopf algebra structure, respectively, is essential to really understanding the Drinfeld double; we have found a counterpart to this idea in the two versions of Yetter-Drinfeld modules in the present paper.

\section{REFERENCES}

1. Dijkgraff, R., Pasquier, V., And Roche, P. Quasi Hopf algebras, group cohomology and orbifold models. Nuclear Phys. B Proc. Suppl. 18B (1990), 60-72. MR 92m:82138

2. Drinfel'D, V. G. Quasi-Hopf algebras. Leningrad Math. J. 1 (1990), 1419-1457. MR 91b:17016

3. Hausser, F., AND Nill, F. Diagonal crossed products by duals of quasi-quantum groups. Rev. Math. Phys. 11 (1999), 553-629. MR 2000d:81069

4. Hausser, F., And Nill, F. Doubles of quasi-quantum groups. Comm. Math. Phys. 199 (1999), 547-589. MR 2000a:16075

5. Hausser, F., AND NiLl, F. Integral theory for quasi-Hopf algebras. preprint (math. QA/9904164).

6. Joyal, A., And Street, R. Braided tensor categories. Adv. in Math. 102 (1993), 20-78.

7. Kassel, C. Quantum Groups, vol. 155 of Graduate Texts in Mathematics. Springer, 1995. MR 94m:18008

8. Lyubashenko, V. Hopf algebras and vector symmetries. Russ. Math. Surveys 41 (1988), 153-154. MR 88c:58007

9. MAJID, S. Foundations of quantum group theory. Cambridge Univ. Press, 1995 . MR 97g:17016 
10. MajID, S. Quantum double for quasi-Hopf algebras. Lett. Math. Phys. 45 (1998), 1-9. MR 2000b:16077

11. Pareigis, B. Non-additive ring and module theory I. General theory of monoids. Publ. Math. Debrecen 24 (1977), 189-204. MR 56:8656

12. Pareigis, B. Non-additive ring and module theory II. $\mathcal{C}$-categories, $\mathcal{C}$-functors and $\mathcal{C}$ morphisms. Publ. Math. Debrecen 24 (1977), 351-361. MR 58:16834a

13. Schauenburg, P. Hopf modules and Yetter-Drinfel'd modules. J. Algebra 169 (1994), 874890. MR 95j:16047

14. Schauenburg, P. Hopf algebra extensions and monoidal categories. preprint (2001).

15. Sweedler, M. E. Hopf Algebras. Benjamin, New York, 1969. MR 40:5705

16. Tambara, D. The coendomorphism bialgebra of an algebra. J. Fac. Sci. Univ. Tokyo, Sect.IA, Math. 37 (1990), 425-456. MR 91f:16048

17. Woronowicz, S. L. Differential calculus on compact matrix pseudogroups (quantum groups). Comm. Math. Phys. 122 (1989), 125-170. MR 90g:58010

Mathematisches Institut der Universität München, Theresienstr. 39, 80333 München, GERMANY

E-mail address: schauen@rz.mathematik.uni-muenchen.de 